\title{
Analysis of Factors that Influence Customers' Willingness to Leave Big Data Digital Footprints on Social Media: A Systematic Review of Literature
}

\begin{abstract}
:
Big data has been discussed extensively in existing scholarly works but scant consideration is given to customers' willingness to generate and leave big data digital footprints on social media, especially in the light of the profusely debated issue of privacy and security. The current paper endeavours to address this gap in the literature by developing a conceptual framework. In doing so, this paper conducts a systematic review of extant literature from 2002 to 2017 to identify and analyse the underlying factors that influence customers' willingness to leave digital footprints on social media. The findings of this review reveal that personal behaviour (intrinsic psychological dispositions), technological factors (relative advantage and convenience), social influence (social interaction, social ties and social support) and privacy and security (risk, control and trust) are the key factors that influence customers' willingness to generate and leave big data digital footprints on social media. The conceptual framework presented in this paper advances the scholarship of technology adoption and use and provides useful direction for future empirical research for both academics and practitioners.
\end{abstract}

Key Words: Big Data Digital Footprint; Social Media; Privacy and Security, Technology, Personal Behaviour, Social Influence

Unified Theory of Acceptance and Use of Technology model (UTAUT2), which takes into account various aspects of customers' use of technology to offer deep insights into the dynamics and kinetics of customers' willingness to deposit digital DNA on social media 


\section{Introduction}

Big data digital footprints are digital DNA that customers generate and leave on digital platforms when they interact with and use various media channels, including social media. The ever-increasing use of and interaction with social media has intensified researchers' and practitioners' interest in customers' social media led digital footprints (Rauniar et al., 2013; Tuton and Solomon, 2015). However, research on customers' perceptions of and attitude towards generating big social data digital footprints is still in its infancy.

Digital footprints are described as social data created by customers when they interact with media channels. Such digital footprints are not just identities but also memories, moments and behaviour. Social media providers who collect these huge digital chronicles can determine how and why users behave and purchase on digital platforms (Fish, 2009). Social media use has grown exponentially and has become an integral part of consumer life. With the advent of Web 2.0, digital footprint generation has increased significantly. It is estimated that 44 times more data generation would take place, from 2009 to 44 zettabytes of data by 2020 (CSC 2017). In addition, the exponential growth of mobile telephony (Sharma, 2017), cloud computing and $4 \mathrm{G}$ networks have created many more social media touchpoints. As a result, customers are found to be connected to smart devices (smartphones, tablets, smartwatches, Cortana, Siri and Alexa etc.) 24/7, generating and leaving behind huge digital trails for service providers. Furthermore, user-generated content on social media will be the main channel for the enrichment of information base for public administrative bodies and commercial firms (Baur, 2017).

Tuton and Solomon (2015) divided the use of social media into four zones based on different social media channels and vehicles: i.e. social community interaction, social commerce, social publishing and social entertainment. Customers create their digital DNA on channels in each of these zones by sharing comments, photos, videos, blogs, bookmarks, reviews, ratings and social shopping, linking with government applications etc. (Malhotra et al., 2012; Rosenberger et al., 2017). These digital trails exhibit their interests, social and cultural identities, and occupational and geographical attachments, which are essentially required by firms (Charlesworth, 2014; Michael et al., 2014). Moreover, these digital traces help firms to analyse customers' sentiments and contents by using advanced analytics to gain deeper insight into their behaviour and develop their profiles (Charlesworth, 2014; DWork and Mulligan, 2013).

Customers use social media excessively, but they may or may not be aware of the digital footprints that they leave for companies such as Google, Yahoo, Amazon and Facebook. The services of social media platforms (Facebook, Twitter and Instagram etc.) have redefined the ways in which business value can be generated, as these providers and tech giants use personal data to track customers and their behaviour through invasive and ubiquitous crawling. They use algorithms to generate powerful insight through data connections, inferences and data interpretations (DWork and Mulligan, 2013). Furthermore, as the competition amongst firms is increasing to seek innovative capabilities to mine digital trails and gain a competitive edge over rivals. Hence, the managerial implications for big data digital footprints are immense, as they can create value and promote development (Pulse, 2012).

On the other hand, the pervasive use of digital footprints has raised privacy and security concerns amongst social media users. This area is of huge interest for individuals, public and 
governments as to where to draw the line for privacy and security of unauthorised access to individual digital footprints. This has even led many countries to initiate measures to protect individual privacy and security as social media platforms and web technologies have become more pervasive and vulnerable to abuse and exploitation. Recently in the USA, privacy advocates allege that large Internet service providers can potentially encroach to consumers' privacy as they have access to large volume of personal data (Waters and Bond, 2017). Even everyday objects, connected to the Internet, are collecting personal digital footprints (Kuchler, 2017).

Although consumers' engagement with social media has received significant research attention (Al-Jabri et al., 2015; Charlesworth, 2014; Hajli, 2014; Hsu and Wu, 2011; Akar and Topçu 2011; Hau and Kim, 2010), there is paucity of research that identifies and analyses the factors that influence customers' intention to generate and leave big data digital footprints on social media. Hence, further investigation will not only contribute to big data and social media literature, but will also advance privacy and security scholarship. Our study addresses this research gap by identifying and analysing the factors that determine customers' willingness to generate and leave digital DNAs on social media and how privacy and security deter or facilitate digital footprint generation on social media.

Furthermore, the current literature provides the theoretical framework on customers use and adoption of social media (Hsu and Wu, 2011; Lin and Anol, 2008; Lu et al., 2009; Venkatesh et al., 2012; Wei et al., 2009), for instance, Technology Acceptance Model (TAM), Theory of Reasoned Action (TRA), Theory of Planned Behaviour (TPB), Uses and Gratifications (U\&G) and the Unified Theory of Acceptance and Use of Technology (UTAUT1 and UTAUT2) etc. are widely cited theories and frameworks for assessing customers' use of social media. These theories take into account factors that influence users' acceptance and use of social media such as usefulness, relative advantage, ease of use, innovation, social and compatibility issues (Al-Gahtani et al., 2007; Davis, 1989; Davis et al., 1989; Hsu and Wu, 2011; Lin and Anol, 2008; Lu et al., 2009; Venkatesh et al., 2003; Venkatesh et al., 2012; Wei et al., 2009). However they do not fully capture the dynamics and kinetics of customers' willingness to generate and leave big data digital footprints on social media and provide limited scope for generalisation. Furthermore, they do not address how privacy and security affect customers' willingness to generate digital footprint on social media. The current paper aims to develop appropriate conceptual scaffoldings for customers' willingness to generate and leave big data digital footprints on social media by critically examining and synthesising the above mentioned theories and concepts that have been extensively cited and applied in information systems, management and marketing literature.

Therefore, we feel there is a need for a conceptual paper to develop a theoretical framework through a rigorous and systematic review of existing academic literature (Cropanzano, 2009). A conceptual paper will offer theoretical impetus for future scholarly works and a theoretical framework for empirical investigation. Hence, the current paper aims to present a conceptual framework that defines the nascent and potential inter-relationships amongst various constructs.

The paper is structured as follows: The first section describes the methodology of the study. The next section delineates the findings and analysis. The final section discusses the results and their theoretical and practical implications, along with limitations and future research direction. 


\section{Methodology of the Systematic Review}

In order to study customers' willingness to generate and leave digital footprints on social media, a desk-based study was carried out based on the approach of Ngai et al. (2009) and Ngai and Wat (2002) as follows.

\section{Classification Process}

The following steps were carried out in the systematic review.

1. Database search

2. First classification

3. Verification of first classification

4. Final verification

The literature around the use of social media and big data digital footprint was found in a number of different journals belonging to a wide range of disciplinary origins. The following online journal databases were used to identify published peer reviewed articles: EBSCO Host, Emerald, IEEE/IET, Electronic Library, ProQuest, Sage, Science Direct and Scopus.

The following criteria were used to screen and classify the articles. Only peer reviewed articles were chosen to represent advanced research output as suggested by Ngai et al (2009). Articles that have main themes around the use of social media, big data digital footprint, customers' digital footprint, factors influencing the use of social media were chosen. In order to carry out a thorough desk based systematic review, the following appropriate search terms were identified after detailed discussions amongst the authors based on the literature review, following Nill and Schibrowsky (2007). Furthermore, they were searched individually through the search algorithm on the chosen databases. They were rephrased and cross checked to ensure articles fall into the area of the research as the extent literature suggests. "Customers' digital footprint on social media", "Why customers leave digital footprint on social media", "Big data digital footprint of customers on social media", "Customers' use of social media", "Why customers generate digital footprint on social media". Based on the chosen approach, only peer-reviewed journal articles were selected. The period from January 2002 to May 2017 was selected considering the topicality of the issue.

The literature review resulted initially in the identification of 506 articles. The full text of each article was reviewed by the first and second authors, confirmed through discussion and verified by the third author. Feedback from two other independent academics was collected to ensure the validity and reliability of the process. A classification framework, shown in Figure 1 , was developed based on the key emerging factors from the systematic review along with their sub-factors. These four key emerging themes were found to be the leading factors determining customers' use of social media and their digital footprint generation. As a result, based on these four key factors, 58 articles were selected from the initial sample of 501 articles, although this selection was not exhaustive. 


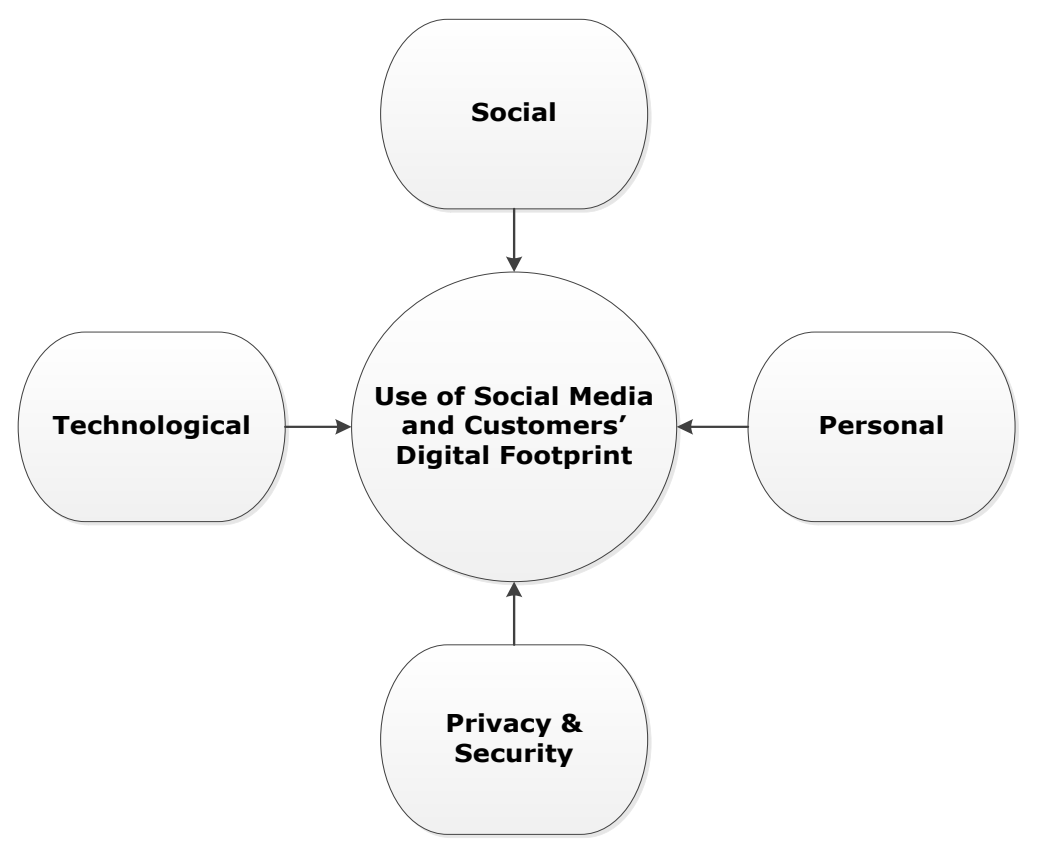

Fig.1 Classification Framework

\section{Data Reporting}

A six-column table (Table 1) was designed to report data captured from the final 58 articles. It was used to examine the constructs used by the previous studies to determine customers' use and willingness to generate and leave big data digital footprints on social media. The following table delineates the details of constructs identified in each study, along with the choice of theories, research approach, design and findings respectively.

Table 1: Summary of Systematic Review of Previous Scholarly Works

\begin{tabular}{|c|c|c|c|c|c|}
\hline No & Reference & Theory & Constructs & $\begin{array}{c}\text { Research } \\
\text { Approach } \\
\text { and Design }\end{array}$ & Research Finding \\
\hline 1 & $\begin{array}{l}\text { Whiting and } \\
\text { Williams (2013) }\end{array}$ & $\begin{array}{l}\text { Uses and Gratifications } \\
\text { (UandG) }\end{array}$ & $\begin{array}{l}\text { Entertainment, relaxation, convenience, } \\
\text { information seeking, social interaction }\end{array}$ & $\begin{array}{l}\text { Qualitative, } \\
\text { interviews }\end{array}$ & $\begin{array}{l}\text { Uses and gratifications theory has } \\
\text { specific relevance and therefore should } \\
\text { have prominence in social media } \\
\text { marketing. }\end{array}$ \\
\hline 2 & $\begin{array}{l}\text { Idemudia et al. } \\
\text { (2016) }\end{array}$ & $\begin{array}{l}\text { Technology Acceptance } \\
\text { Model (TAM), Diffusion } \\
\text { of Innovation }\end{array}$ & $\begin{array}{l}\text { Ease of use, usefulness, satisfaction, } \\
\text { relative advantage, compatibility, } \\
\text { information quality, and risk }\end{array}$ & $\begin{array}{l}\text { Quantitative } \\
\text { and a survey }\end{array}$ & $\begin{array}{l}\text { Ease of use, usefulness, and satisfaction } \\
\text { of social media have a positive and } \\
\text { significant influence on social media } \\
\text { continuance usage. }\end{array}$ \\
\hline 3 & $\begin{array}{l}\text { Ngai et al. } \\
\text { (2015) }\end{array}$ & $\begin{array}{l}\text { Technology Acceptance } \\
\text { Model (TAM), Theory of } \\
\text { Reasoned Action (TRA), } \\
\text { Theory of Planned } \\
\text { Behaviour (TPB) and } \\
\text { UandG }\end{array}$ & $\begin{array}{l}\text { Personality traits, role of technology, } \\
\text { social capital and gratification }\end{array}$ & $\begin{array}{l}\text { Qualitative, } \\
\text { critical } \\
\text { literature } \\
\text { review and a } \\
\text { case study }\end{array}$ & $\begin{array}{l}\text { Social media applications are supported } \\
\text { by social media technologies and tools. }\end{array}$ \\
\hline 4 & $\begin{array}{l}\text { Gamboa and } \\
\text { Gonçalves } \\
\text { (2014) }\end{array}$ & $\mathrm{X}$ & $\begin{array}{l}\text { Trust, customer satisfaction, perceived } \\
\text { value, } \\
\text { and commitment }\end{array}$ & $\begin{array}{l}\text { Quantitative } \\
\text { and a survey }\end{array}$ & $\begin{array}{l}\text { Facebook enhances relations that } \\
\text { increase customer loyalty through trust, } \\
\text { commitment, perceived value and } \\
\text { customer satisfaction. }\end{array}$ \\
\hline 5 & $\begin{array}{l}\text { Akar and Topçu } \\
\text { (2011) }\end{array}$ & $\mathrm{X}$ & $\begin{array}{l}\text { Use, attitude, fear, knowledge, } \\
\text { monitoring, foresight, security and } \\
\text { privacy }\end{array}$ & $\begin{array}{l}\text { Quantitative } \\
\text { and a survey }\end{array}$ & $\begin{array}{l}\text { Consumer behaviour in the virtual } \\
\text { world is affected by factors of security } \\
\text { and privacy. }\end{array}$ \\
\hline 6 & Cao et al. & Social capital theory & Trust, Knowledge integration, & Quantitative & Social media have the potential to \\
\hline
\end{tabular}




\begin{tabular}{|c|c|c|c|c|c|}
\hline & (2015) & & Experience, shared language & and a survey & $\begin{array}{l}\text { facilitate the formation of employees' } \\
\text { social capital indicated by social } \\
\text { networking, trust and shared language. }\end{array}$ \\
\hline 7 & $\begin{array}{l}\text { De Valck et al. } \\
(2009)\end{array}$ & $\begin{array}{l}\text { Theories of interpersonal } \\
\text { influence, reference group } \\
\text { influence, word of mouth }\end{array}$ & Interaction, social ties, need recognition & $\begin{array}{l}\text { Quantitative } \\
\text { and a survey } \\
\text { (netnography) }\end{array}$ & $\begin{array}{l}\text { Consumer decision-making process is } \\
\text { affected by interaction, social ties, need } \\
\text { recognition and post-purchase } \\
\text { evaluation. }\end{array}$ \\
\hline 8 & Hajli (2014) & TAM & Trust, perceived usefulness & $\begin{array}{l}\text { Quantitative } \\
\text { and a survey }\end{array}$ & $\begin{array}{l}\text { Social interactions on social media } \\
\text { enhance consumers' trust, affecting } \\
\text { buying intention and perceived } \\
\text { usefulness. }\end{array}$ \\
\hline 9 & $\begin{array}{l}\text { Pentina et al. } \\
\text { (2013) }\end{array}$ & $X$ & $\begin{array}{l}\text { Trust, perceived personality and } \\
\text { patronage intention (visit websites, } \\
\text { purchase and recommend to others) }\end{array}$ & $\begin{array}{l}\text { Quantitative } \\
\text { and a survey }\end{array}$ & $\begin{array}{l}\text { Trust in social media brands has a } \\
\text { positive relationship with patronage } \\
\text { intention. }\end{array}$ \\
\hline 10 & $\begin{array}{l}\text { Hsiao et al. } \\
(2010)\end{array}$ & $\mathrm{X}$ & $\begin{array}{l}\text { Perceived ability, perceived critical } \\
\text { mass, perceived benevolence and trust }\end{array}$ & $\begin{array}{l}\text { Quantitative } \\
\text { and a survey }\end{array}$ & $\begin{array}{l}\text { Perceived ability, critical mass, } \\
\text { benevolence, integrity and trust are } \\
\text { important antecedents of trust in } \\
\text { product recommendation on social } \\
\text { networking sites. }\end{array}$ \\
\hline 11 & $\begin{array}{l}\text { Hau and Kim } \\
(2010)\end{array}$ & TPB and ERG & $\begin{array}{l}\text { Intrinsic motivation, shared goals, social } \\
\text { trust, extrinsic motivation and social ties }\end{array}$ & $\begin{array}{l}\text { Quantitative } \\
\text { and a survey }\end{array}$ & $\begin{array}{l}\text { Shared goals and shared trust motivate } \\
\text { in sharing knowledge on social media. }\end{array}$ \\
\hline 12 & Hussain (2012) & $\mathrm{X}$ & $\begin{array}{l}\text { Learning, networking, events, enjoying, } \\
\text { killing time, friending, getting } \\
\text { information }\end{array}$ & $\begin{array}{l}\text { Quantitative } \\
\text { and a survey }\end{array}$ & $\begin{array}{l}\text { Social media is used for educational } \\
\text { and academic purposes to connect and } \\
\text { collaborate with the virtual educational } \\
\text { community. }\end{array}$ \\
\hline 13 & $\begin{array}{l}\text { Hudson and } \\
\text { Hudson (2013) }\end{array}$ & $\mathrm{X}$ & $\begin{array}{l}\text { Consumer decision journey (consider, } \\
\text { evaluate, buy and enjoy, advocate and } \\
\text { bond) }\end{array}$ & $\begin{array}{l}\text { Multi-method } \\
\text { approach and } \\
\text { a case study } \\
\text { methodology }\end{array}$ & $\begin{array}{l}\text { Instead of the traditional purchase } \\
\text { funnel process, customers research } \\
\text { products at the evaluation and post- } \\
\text { purchase stages and enter into an open } \\
\text { relationship with the brand on social } \\
\text { media. }\end{array}$ \\
\hline 14 & $\begin{array}{l}\text { Milewicz and } \\
\text { Saxby (2013) }\end{array}$ & $\mathrm{X}$ & $\begin{array}{l}\text { Perceived social media usefulness, ease } \\
\text { of use, satisfaction, perceived social } \\
\text { pressure, usage intention }\end{array}$ & $\begin{array}{l}\text { Quantitative } \\
\text { and a survey }\end{array}$ & $\begin{array}{l}\text { Perceived social media usefulness and } \\
\text { ease of use influence customer } \\
\text { satisfaction and social media usage } \\
\text { intention. }\end{array}$ \\
\hline 15 & $\begin{array}{l}\text { Hsu and Wu } \\
\text { (2011) }\end{array}$ & UTAUT, EDM and Flow & $\begin{array}{l}\text { Social influence, performance } \\
\text { expectancy, flow experience, effort } \\
\text { expectancy and satisfaction }\end{array}$ & $\begin{array}{l}\text { Quantitative } \\
\text { and a survey }\end{array}$ & $\begin{array}{l}\text { Continuance usage of Facebook is } \\
\text { determined by social influence, } \\
\text { performance expectancy, flow } \\
\text { experience, effort expectancy and } \\
\text { satisfaction. }\end{array}$ \\
\hline 16 & $\begin{array}{l}\text { Pereira et al. } \\
(2014)\end{array}$ & $\mathrm{X}$ & $\begin{array}{l}\text { Trust, contests and promotions, } \\
\text { experience with the brand, follow } \\
\text { friends }\end{array}$ & $\begin{array}{l}\text { Quantitative } \\
\text { and a survey } \\
\text { (multi-method } \\
\text { data } \\
\text { collection) }\end{array}$ & $\begin{array}{l}\text { Content affinity, fun, alert to bad } \\
\text { experiences, incentives, help for friends } \\
\text { and recognition are the motivators to } \\
\text { share brand content on Facebook. }\end{array}$ \\
\hline 17 & Ali (2011) & $\begin{array}{l}\text { Social networks and social } \\
\text { capital }\end{array}$ & Obligations, sacrifice, and motivations & $\begin{array}{l}\text { Qualitative } \\
\text { and interviews }\end{array}$ & $\begin{array}{l}\text { Social media generate social support for } \\
\text { consumers and obligations, sacrifice, } \\
\text { and motivations influence the level of } \\
\text { support they receive from social } \\
\text { networks. }\end{array}$ \\
\hline 18 & $\begin{array}{l}\text { Chen and } \\
\text { Sharma (2013) }\end{array}$ & Social Capital Theory & $\begin{array}{l}\text { trust, reciprocity, identification and self- } \\
\text { Disclosure }\end{array}$ & $\begin{array}{l}\text { Quantitative } \\
\text { and a survey }\end{array}$ & $\begin{array}{l}\text { Self-disclosure enables member } \\
\text { interactions, service customizations, } \\
\text { and digital content generation. }\end{array}$ \\
\hline 19 & $\begin{array}{l}\text { Wu et al. } \\
\text { (2010) }\end{array}$ & $\begin{array}{l}\text { Theory of relationship } \\
\text { marketing }\end{array}$ & $\begin{array}{l}\text { Shared values, privacy policy, } \\
\text { satisfaction, trusting belief, } \\
\text { benevolence, integrity, commitment and } \\
\text { stickiness }\end{array}$ & $\begin{array}{l}\text { Quantitative } \\
\text { and a survey }\end{array}$ & $\begin{array}{l}\text { Shared values, satisfaction with } \\
\text { previous interaction and website } \\
\text { privacy policy enhance users' trust. }\end{array}$ \\
\hline 20 & Lu et al. (2010) & $\mathrm{X}$ & $\begin{array}{l}\text { Knowledge, characteristics, institution } \\
\text { and personality-based trust, integrity, } \\
\text { benevolence, ability }\end{array}$ & $\begin{array}{l}\text { Quantitative } \\
\text { and a survey }\end{array}$ & $\begin{array}{l}\text { Trust propensity, perceived similarity, } \\
\text { and structural assurance in members of } \\
\text { virtual community and trust in } \\
\text { members' integrity and benevolence } \\
\text { affect purchase intention. }\end{array}$ \\
\hline 21 & $\begin{array}{l}\text { Liang et al. } \\
\text { (2011) }\end{array}$ & TRA and TPB & $\begin{array}{l}\text { Social support, relationship quality, } \\
\text { website quality }\end{array}$ & $\begin{array}{l}\text { Quantitative } \\
\text { and a survey }\end{array}$ & $\begin{array}{l}\text { Social support (emotional and } \\
\text { informational) and relationship quality } \\
\text { (trust, commitment and satisfaction) } \\
\text { influence users' intention on social } \\
\text { networking sites. }\end{array}$ \\
\hline
\end{tabular}




\begin{tabular}{|c|c|c|c|c|c|}
\hline 22 & $\begin{array}{l}\text { Wang and Wei } \\
(2012)\end{array}$ & $\begin{array}{l}\text { Consumer socialisation } \\
\text { and peer communication }\end{array}$ & $\begin{array}{l}\text { Tie strength, identification and } \\
\text { communication with peers }\end{array}$ & $\begin{array}{l}\text { Quantitative } \\
\text { and a survey }\end{array}$ & $\begin{array}{l}\text { Peer communication at peer group } \\
\text { level, individual tie strength and group } \\
\text { identification positively affect purchase } \\
\text { intention and product attitude on social } \\
\text { media. }\end{array}$ \\
\hline 23 & $\begin{array}{l}\text { Hsu et al. } \\
\text { (2007) }\end{array}$ & Social Cognitive Theory & $\begin{array}{l}\text { Economy, information and identification } \\
\text { based trusts, knowledge sharing, self- } \\
\text { efficacy, personal outcome expectation } \\
\text { and community related outcome } \\
\text { expectation }\end{array}$ & $\begin{array}{l}\text { Quantitative } \\
\text { and a survey }\end{array}$ & $\begin{array}{l}\text { Self-efficacy and outcome expectation } \\
\text { for personal influences and } \\
\text { multidimensional trust support } \\
\text { knowledge sharing in virtual } \\
\text { communities. }\end{array}$ \\
\hline 24 & $\begin{array}{l}\text { Park and Kim } \\
\text { (2014) }\end{array}$ & $\mathrm{X}$ & $\begin{array}{l}\text { Experiential and functional benefits, } \\
\text { quality and perceived relationship } \\
\text { investment }\end{array}$ & $\begin{array}{l}\text { Mixed } \\
\text { method: } \\
\text { netnography } \\
\text { and a survey }\end{array}$ & $\begin{array}{l}\text { Perceived utilitarian benefits and } \\
\text { experiential benefits of social } \\
\text { networking brand's website affect } \\
\text { customers' relationship with the brand. }\end{array}$ \\
\hline 25 & $\begin{array}{l}\text { Domina et al. } \\
\text { (2012) }\end{array}$ & Flow theory and TAM & $\begin{array}{l}\text { Novelty seeking, independent } \\
\text { judgement making, control, enjoyment } \\
\text { and ease of use }\end{array}$ & $\begin{array}{l}\text { Quantitative } \\
\text { and a survey }\end{array}$ & $\begin{array}{l}\text { Perceived enjoyment and control } \\
\text { influence buying intention to shop in } \\
\text { the virtual world and independent } \\
\text { judgement making has a positive } \\
\text { impact on perceived control, ease of use } \\
\text { and enjoyment. }\end{array}$ \\
\hline 26 & $\begin{array}{l}\text { Chen et al. } \\
\text { (2014) }\end{array}$ & $\begin{array}{l}\text { Social networks and social } \\
\text { capital }\end{array}$ & $\begin{array}{l}\text { Openness to change (self-direction, } \\
\text { stimulation), self-transcendence } \\
\text { (benevolence), conservation (security } \\
\text { and conformity tradition), self- } \\
\text { enhancement (power and achievement) }\end{array}$ & $\begin{array}{l}\text { Quantitative } \\
\text { and a survey }\end{array}$ & $\begin{array}{l}\text { Universalism, benevolence, self } \\
\text { enhancement, conservation, openness to } \\
\text { change and hedonism affect users' } \\
\text { motivation for the use of social media. }\end{array}$ \\
\hline 27 & $\begin{array}{l}\text { Yulihasri et al. } \\
\text { (2011) }\end{array}$ & TAM, TRA and TPB & $\begin{array}{l}\text { Compatibility, privacy, security, self- } \\
\text { efficacy, ease of use, usefulness, } \\
\text { normative beliefs }\end{array}$ & $\begin{array}{l}\text { Quantitative } \\
\text { and a survey }\end{array}$ & $\begin{array}{l}\text { In addition to ease of use and } \\
\text { usefulness, students' attitude to online } \\
\text { shopping is influenced by } \\
\text { compatibility, privacy, security and } \\
\text { self-efficacy. }\end{array}$ \\
\hline 28 & $\begin{array}{l}\text { Diffley et al. } \\
\text { (2011) }\end{array}$ & $\mathrm{X}$ & $\begin{array}{l}\text { Communication and relationship } \\
\text { maintenance, strong and weak ties }\end{array}$ & $\begin{array}{l}\text { Qualitative } \\
\text { and a focus } \\
\text { group }\end{array}$ & $\begin{array}{l}\text { Friends, communication and } \\
\text { empowering or giving control to } \\
\text { consumers has a positive effect on their } \\
\text { attitude. }\end{array}$ \\
\hline 29 & $\begin{array}{l}\text { Pillai and } \\
\text { Mukherjee } \\
\text { (2011) }\end{array}$ & TAM & $\begin{array}{l}\text { Perceived } \\
\text { playfulness, usefulness and ease of use } \\
\text { and attitude }\end{array}$ & $\begin{array}{l}\text { Quantitative } \\
\text { and a survey }\end{array}$ & $\begin{array}{l}\text { Perceived enjoyment has a significant } \\
\text { impact on hedonic needs and perceived } \\
\text { usefulness in utilitarian benefits of } \\
\text { websites. }\end{array}$ \\
\hline 30 & $\begin{array}{l}\text { Leung et al. } \\
(2015)\end{array}$ & $\begin{array}{l}\text { Integrating the attitude- } \\
\text { toward-the-ad (Aad) model }\end{array}$ & Experience, attitude, brand cognition & $\begin{array}{l}\text { Quantitative } \\
\text { and a survey }\end{array}$ & $\begin{array}{l}\text { Social media experiences influence } \\
\text { customers' attitude towards social } \\
\text { media sites and in turn influence their } \\
\text { attitude toward the brand. }\end{array}$ \\
\hline 31 & $\begin{array}{l}\text { Kennedy et al. } \\
\text { (2015) }\end{array}$ & $\mathrm{X}$ & $\begin{array}{l}\text { Fairness, contextual integrity, social } \\
\text { justice and well-being }\end{array}$ & $\begin{array}{l}\text { Qualitative } \\
\text { and a focus } \\
\text { group }\end{array}$ & $\begin{array}{l}\text { Beyond privacy and surveillance, the } \\
\text { users identified a concern for fairness } \\
\text { contextual integrity in practice and as a } \\
\text { broader concern about social justice and } \\
\text { well-being. }\end{array}$ \\
\hline 32 & $\begin{array}{l}\text { Ghosh et al. } \\
\text { (2014) }\end{array}$ & eWOM & $\begin{array}{l}\text { Perceived product knowledge, source } \\
\text { credibility, involvement level, perceived } \\
\text { risk, brand attitude, intention }\end{array}$ & $\begin{array}{l}\text { Qualitative } \\
\text { literature } \\
\text { review and a } \\
\text { conceptual } \\
\text { model }\end{array}$ & $\begin{array}{l}\text { Perceived risk mediates the relationship } \\
\text { of WOM and brand attitude, and brand } \\
\text { attitude mediates the relationship } \\
\text { between purchase intention and } \\
\text { perceived risk }\end{array}$ \\
\hline 33 & $\begin{array}{l}\text { Kim et al. } \\
(2011)\end{array}$ & $\begin{array}{l}\text { Customer value theory } \\
\text { (Functional, emotional, } \\
\text { social) }\end{array}$ & $\begin{array}{l}\text { Functional (price utility, functional } \\
\text { quality) emotional (aesthetics, } \\
\text { playfulness), social (social self-image } \\
\text { expression, social relationship support) }\end{array}$ & $\begin{array}{l}\text { Quantitative } \\
\text { and a survey }\end{array}$ & $\begin{array}{l}\text { Emotional and social dimension has a } \\
\text { more significant effect on purchase } \\
\text { intention in the sale of digital items to } \\
\text { social networking community than } \\
\text { functional values. }\end{array}$ \\
\hline 34 & $\begin{array}{l}\text { Zhang et al. } \\
\text { (2014) }\end{array}$ & SOR & $\begin{array}{l}\text { Perceived interactivity, personalization } \\
\text { and sociability, social support, presence } \\
\text { and flow }\end{array}$ & $\begin{array}{l}\text { Quantitative } \\
\text { and a survey }\end{array}$ & $\begin{array}{l}\text { Intention is influenced by social } \\
\text { presence, social support and flow. }\end{array}$ \\
\hline 35 & $\begin{array}{l}\text { Zolkepli and } \\
\text { Kamarulzaman } \\
\text { (2015) }\end{array}$ & UandG & $\begin{array}{l}\text { Need category, personal (enjoyment and } \\
\text { entertainment), social (social interaction } \\
\text { and influence) and tension release } \\
\text { (playfulness, companionship and } \\
\text { belongingness), innovation } \\
\text { (compatibility, observability and relative } \\
\text { advantage). }\end{array}$ & $\begin{array}{l}\text { Mixed } \\
\text { method: focus } \\
\text { group (in- } \\
\text { depth } \\
\text { interview) and } \\
\text { a survey }\end{array}$ & $\begin{array}{l}\text { Three types of need category -personal } \\
\text { (enjoyment and entertainment), social } \\
\text { (social interaction and influence) and } \\
\text { tension release (playfulness, } \\
\text { companionship and belongingness) - } \\
\text { drive social media adoption. These } \\
\text { needs are in turn driven by social media }\end{array}$ \\
\hline
\end{tabular}




\begin{tabular}{|c|c|c|c|c|c|}
\hline & & & & & $\begin{array}{l}\text { innovation (compatibility, observability } \\
\text { and relative advantage). }\end{array}$ \\
\hline 36 & $\begin{array}{l}\text { Jansen et al. } \\
(2009)\end{array}$ & eWOM & $\begin{array}{l}\text { Brand Knowledge (brand awareness, } \\
\text { brand image), brand relationship (brand } \\
\text { satisfaction and trust) brand attachment }\end{array}$ & $\begin{array}{l}\text { Collected } \\
\text { tweets on } \\
\text { Twitter }\end{array}$ & $\begin{array}{l}\text { Web communications and social } \\
\text { networking services influence } \\
\text { consumers' brand perception and } \\
\text { purchasing decisions. }\end{array}$ \\
\hline 37 & $\begin{array}{l}\text { Bharati et al. } \\
\text { (2014) }\end{array}$ & $\begin{array}{l}\text { Organisational innovation } \\
\text { and institutional theory }\end{array}$ & $\begin{array}{l}\text { Normative pressure, coercive pressure } \\
\text { and absorptive capacity }\end{array}$ & $\begin{array}{l}\text { Quantitative } \\
\text { and a survey }\end{array}$ & $\begin{array}{l}\text { Institutional pressures have no direct } \\
\text { effect on social media assimilation. }\end{array}$ \\
\hline 38 & $\begin{array}{l}\text { Chow and Shi } \\
\text { (2015) }\end{array}$ & Customer value theory & $\begin{array}{l}\text { Functional value (information quality, } \\
\text { product related learning, economic } \\
\text { benefit), social value (interactivity, } \\
\text { collaboration, social presence), } \\
\text { emotional value (entertainment, arousal) }\end{array}$ & $\begin{array}{l}\text { Quantitative } \\
\text { and a survey }\end{array}$ & $\begin{array}{l}\text { Multidimensional customer values } \\
\text { enhance companies' use of brand } \\
\text { pages' satisfaction on SNS. }\end{array}$ \\
\hline 39 & $\begin{array}{l}\text { Foster et al. } \\
(2011)\end{array}$ & $\mathrm{X}$ & $\begin{array}{l}\text { Information seeking, socialisation and } \\
\text { content creation }\end{array}$ & $\begin{array}{l}\text { Quantitative } \\
\text { and a survey }\end{array}$ & $\begin{array}{l}\text { Users can be segmented } \\
\text { heterogeneously based on their need for } \\
\text { information seeking, socialisation and } \\
\text { content creation. }\end{array}$ \\
\hline 40 & $\begin{array}{l}\text { Al-Jabri et al. } \\
\text { (2015) }\end{array}$ & UandG & $\begin{array}{l}\text { Self-presentation, social interaction, } \\
\text { freedom of expression, enjoyment, } \\
\text { gender and user experience, education } \\
\text { and income }\end{array}$ & $\begin{array}{l}\text { Quantitative } \\
\text { and a survey }\end{array}$ & $\begin{array}{l}\text { Freedom of expression, social } \\
\text { interaction and enjoyment influence the } \\
\text { usage of Twitter. }\end{array}$ \\
\hline 41 & $\begin{array}{l}\text { Ellison et al. } \\
(2007)\end{array}$ & Social Capital & Types of social capital & $\begin{array}{l}\text { Quantitative } \\
\text { and a survey }\end{array}$ & $\begin{array}{l}\text { Strong association between the use of } \\
\text { social networking sites and the three } \\
\text { types of social capital, namely bridging, } \\
\text { bonding and maintaining, along with } \\
\text { psychological well-being, self-esteem } \\
\text { and satisfaction with life. }\end{array}$ \\
\hline 42 & $\begin{array}{l}\text { Grace et al. } \\
(2015)\end{array}$ & $\begin{array}{l}\text { Stimuli Organism } \\
\text { Response, Cognitive- } \\
\text { affective system theory of } \\
\text { personality }\end{array}$ & $\begin{array}{l}\text { Perceived psychological features (self- } \\
\text { image control risk, interaction and } \\
\text { usage volition) }\end{array}$ & $\begin{array}{l}\text { Quantitative } \\
\text { and a survey }\end{array}$ & $\begin{array}{l}\text { Perceived psychological features of } \\
\text { Facebook as self-image control risk, } \\
\text { interaction and usage volition determine } \\
\text { context relevant psychological } \\
\text { dispositions to predict Facebook usage } \\
\text { and non-usage behaviour. }\end{array}$ \\
\hline 43 & $\begin{array}{l}\text { Krasonikolakis } \\
\text { et al. (2014) }\end{array}$ & $\begin{array}{l}\text { Virtual Worlds (VWs), } \\
\text { Virtual reality retailing } \\
\text { (VRR) }\end{array}$ & $\begin{array}{l}\text { Security and privacy and core store } \\
\text { features }\end{array}$ & $\begin{array}{l}\text { Mixed } \\
\text { method: } \\
\text { survey and in- } \\
\text { depth } \\
\text { interviews }\end{array}$ & $\begin{array}{l}\text { Consumer behaviour in the virtual } \\
\text { world (Second Life) was affected by } \\
\text { two key factors of security and privacy } \\
\text { and core store features }\end{array}$ \\
\hline 44 & $\begin{array}{l}\text { Chang and } \\
\text { Chuang (2011) }\end{array}$ & Social capital & $\begin{array}{l}\text { Language of users, altruism, reciprocity } \\
\text { and identification, reputation, trust and } \\
\text { social interaction }\end{array}$ & $\begin{array}{l}\text { Quantitative } \\
\text { and a survey }\end{array}$ & $\begin{array}{l}\text { Shared language of users, altruism, } \\
\text { reciprocity and identification enhance } \\
\text { knowledge sharing behaviour on social } \\
\text { media. }\end{array}$ \\
\hline 45 & $\begin{array}{l}\text { Chen et al. } \\
\text { (2014). }\end{array}$ & Commitment theory & $\begin{array}{l}\text { Effective commitment (emotional } \\
\text { attachment), continuance commitment } \\
\text { (cost of switching too high) and } \\
\text { normative commitment }\end{array}$ & $\begin{array}{l}\text { Mixed } \\
\text { method: } \\
\text { Delphi } \\
\text { method and a } \\
\text { survey }\end{array}$ & $\begin{array}{l}\text { Effective commitment and continuance } \\
\text { commitment are good predictors of } \\
\text { active behaviour on social media. }\end{array}$ \\
\hline 46 & $\begin{array}{l}\text { Gironda and } \\
\text { Korgaonkar } \\
\text { (2014) }\end{array}$ & $\begin{array}{l}\text { TRA, TPB and } \\
\text { Decomposed theory of } \\
\text { planned behaviour (DTPB) }\end{array}$ & $\begin{array}{l}\text { Relative advantage, complexity, } \\
\text { compatibility, attitude and intention, } \\
\text { subjective norms and behaviour control }\end{array}$ & $\begin{array}{l}\text { Quantitative } \\
\text { and a survey }\end{array}$ & $\begin{array}{l}\text { Relative advantage, complexity, } \\
\text { compatibility, normative influences as } \\
\text { antecedents of attitude and intention of } \\
\text { consumer behaviour on social media } \\
\text { along with subjective norms and } \\
\text { behaviour control. }\end{array}$ \\
\hline 47 & Chiang (2013) & $\begin{array}{l}\text { TPB, TAM, TRA, UandG, } \\
\text { Innovation diffusion theory }\end{array}$ & $\begin{array}{l}\text { Complexity, relative advantage, } \\
\text { compatibility, informativeness, social } \\
\text { interactivity, playfulness, attitude, } \\
\text { intention }\end{array}$ & $\begin{array}{l}\text { Quantitative } \\
\text { and a survey }\end{array}$ & $\begin{array}{l}\text { Continuous usage of social media } \\
\text { varied with different innovation } \\
\text { diffusion stages and attitude affects } \\
\text { usage intention }\end{array}$ \\
\hline 48 & $\begin{array}{l}\text { Dennis et al. } \\
\text { (2009) }\end{array}$ & TRA, TAM & $\begin{array}{l}\text { Emotional state, consumer traits, } \\
\text { interactivity, social factors, image and } \\
\text { situational factors (convenience, variety) } \\
\text { influence attitude and trust }\end{array}$ & $\begin{array}{l}\text { Qualitative, } \\
\text { literature } \\
\text { review }\end{array}$ & $\begin{array}{l}\text { Attitude drives e-consumer behaviour } \\
\text { to actual purchase. Emotional state, } \\
\text { consumer traits, interactivity, social } \\
\text { factors, image and situational factors } \\
\text { influence attitude and trust, leading to } \\
\text { intention and actual purchase. }\end{array}$ \\
\hline 49 & $\begin{array}{l}\text { Dalla Pozza } \\
\text { (2014) }\end{array}$ & $\mathrm{X}$ & $\begin{array}{l}\text { Perceived utility, knowledge, social } \\
\text { motivation, past experience, situational } \\
\text { moderators, customer moderators } \\
\text { (personality, demographics), service } \\
\text { quality }\end{array}$ & $\begin{array}{l}\text { Qualitative, } \\
\text { semi- } \\
\text { structured } \\
\text { interviews }\end{array}$ & $\begin{array}{l}\text { Facebook users have social motivation } \\
\text { and Twitter users are driven by } \\
\text { utilitarian motivation }\end{array}$ \\
\hline
\end{tabular}




\begin{tabular}{|c|c|c|c|c|c|}
\hline 50 & $\begin{array}{l}\text { Campbell et al. } \\
\text { (2014). }\end{array}$ & $\mathrm{X}$ & $\begin{array}{l}\text { Psychological, socio-demographic and } \\
\text { economic covariates }\end{array}$ & $\begin{array}{l}\text { Mixed } \\
\text { method: focus } \\
\text { group and a } \\
\text { survey }\end{array}$ & $\begin{array}{l}\text { Consumers' engagement with social } \\
\text { media is based on psychological, socio- } \\
\text { demographic and economic covariates. }\end{array}$ \\
\hline 51 & $\begin{array}{l}\text { Cheung et al. } \\
\text { (2015). }\end{array}$ & $\begin{array}{l}\text { Social exchange and } \\
\text { privacy calculus } \\
\text { theories }\end{array}$ & $\begin{array}{l}\text { Cost mitigating factors (trust in } \\
\text { members and service provider, } \\
\text { perceived control), perceived cost } \\
\text { (perceived privacy risk), perceived } \\
\text { benefits (convenience, new relationship } \\
\text { building, self-presentation, enjoyment, } \\
\text { social influence) }\end{array}$ & $\begin{array}{l}\text { Quantitative } \\
\text { and a survey }\end{array}$ & $\begin{array}{l}\text { Perceived benefits and social influence } \\
\text { have strong effect on self-disclosure on } \\
\text { social media. }\end{array}$ \\
\hline 52 & $\begin{array}{l}\text { Presi et al. } \\
\text { (2014). }\end{array}$ & $\mathrm{X}$ & $\begin{array}{l}\text { Altruism and self-enhancement, } \\
\text { vengeance, venting }\end{array}$ & $\begin{array}{l}\text { Quantitative } \\
\text { and a survey }\end{array}$ & $\begin{array}{l}\text { Social media customers who have } \\
\text { altruistic and self-enhancement } \\
\text { motivators have a positive attitude } \\
\text { towards a firm's response but } \\
\text { customers motivated by vengeance } \\
\text { have a negative attitude towards a } \\
\text { firm's response. }\end{array}$ \\
\hline 53 & $\begin{array}{l}\text { Lambiotte and } \\
\text { Kosinski (2014) }\end{array}$ & $\mathrm{X}$ & $\begin{array}{l}\text { Psychological traits (neuroticism, } \\
\text { extroversion) }\end{array}$ & $\begin{array}{l}\text { Qualitative, } \\
\text { literature } \\
\text { review }\end{array}$ & $\begin{array}{l}\text { How big data digital footprints like } \\
\text { Facebook profiles and mobile device } \\
\text { logs could be used to identify } \\
\text { personalities and predict individuals' } \\
\text { psychological traits from their digital } \\
\text { footprint. }\end{array}$ \\
\hline 54 & $\begin{array}{l}\text { Mathwick } \\
(2002)\end{array}$ & $\mathrm{X}$ & $\begin{array}{l}\text { Behaviour loyalty, effort, enjoyment, } \\
\text { entertainment, escapism }\end{array}$ & $\begin{array}{l}\text { Quantitative } \\
\text { and a survey }\end{array}$ & $\begin{array}{l}\text { Social media consumers are socialisers, } \\
\text { lurkers, transaction community } \\
\text { members and personal connectors based } \\
\text { on their behaviour and relational norms. }\end{array}$ \\
\hline 55 & $\begin{array}{l}\text { Burgess et al. } \\
\text { (2011) }\end{array}$ & $\mathrm{X}$ & Trustworthiness & $\begin{array}{l}\text { Quantitative } \\
\text { and a survey }\end{array}$ & $\begin{array}{l}\text { There are differences in the level of } \\
\text { trust for online travel information from } \\
\text { different sources, greater trust is placed } \\
\text { in online travel comments when they } \\
\text { are on a specific travel website than } \\
\text { when they are on social networking } \\
\text { sites. }\end{array}$ \\
\hline 56 & $\begin{array}{l}\text { Rishika et al. } \\
\text { (2013) }\end{array}$ & $\mathrm{X}$ & $\begin{array}{l}\text { Share experience, voice opinions, gain } \\
\text { benefits and satisfaction. }\end{array}$ & $\begin{array}{l}\text { Mixed } \\
\text { method: } \\
\text { Webpage on } \\
\text { SNS } \\
\text { (Facebook) to } \\
\text { connect with } \\
\text { customers and } \\
\text { a survey }\end{array}$ & $\begin{array}{l}\text { Customers' participation is high when } \\
\text { firms have high media efforts and social } \\
\text { media give customers the opportunity } \\
\text { to share experience, voice opinions, and } \\
\text { gain benefits and satisfaction. }\end{array}$ \\
\hline 57 & Tucker (2014) & 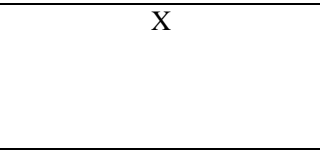 & Privacy control & $\begin{array}{l}\text { Quantitative, } \\
\text { randomized } \\
\text { field } \\
\text { experiment }\end{array}$ & $\begin{array}{l}\text { Social media customers react positively } \\
\text { when web platforms give control to } \\
\text { their users. }\end{array}$ \\
\hline 58 & $\begin{array}{l}\text { Jiang et al } \\
\text { (2013). }\end{array}$ & $\begin{array}{l}\text { Hyperpersonal Framework } \\
\text { and Privacy Calculus }\end{array}$ & $\begin{array}{l}\text { Aspects of hyperpersonal } \\
\text { framework (Perceived anonymity of } \\
\text { self, Perceived anonymity of others, } \\
\text { Perceived media richness, Perceived } \\
\text { intrusiveness), Privacy trade-off } \\
\text { (Privacy concerns, Social rewards), } \\
\text { Privacy-protective behaviour (Self- } \\
\text { disclosure, Misrepresentation) }\end{array}$ & $\begin{array}{l}\text { Quantitative } \\
\text { and a survey }\end{array}$ & $\begin{array}{l}\text { Perceived anonymity and perceived } \\
\text { intrusiveness affect privacy concerns } \\
\text { and social rewards, and individuals } \\
\text { utilise misrepresentation and self- } \\
\text { disclosure to protect privacy. }\end{array}$ \\
\hline
\end{tabular}

\section{Findings and Analysis}

The systematic review of scholarly works led to the development of the above classification framework (Fig. 1) and identification of the key factors. These factors were found to be the leading determinants of the use of social media and customers' digital footprint generation. Further analyses of each of the factor along with some sub-factors are provided below. 


\section{Personal behaviour}

Personal behaviour consists of personal intrinsic and extrinsic motivation (sub-factors of personal behaviour are listed in Figure 2). It has been found that the use of social media is mostly influenced by intrinsic motivation such as intrinsic perceived functional and emotional benefits; self-enhancement, self-esteem and ego (Diffley et al., 2011; Hau and Kim, 2011). Users are driven by their perceived intrinsic experiential benefits, sensory pleasure (hedonic and emotional: Park and Kim, 2014) and self-enhancement, which satisfy their hedonic needs and extrinsic benefits. Similar findings are noted by Whiting and Williams (2013), who report that users tend to engage with social media to fulfil their intrinsic psychological needs of entertainment, relaxation and expression of opinions. The extant literature suggests that psychological intrinsic emotional factors of enjoyment, pleasure, and self-enhancement affect social media user behaviour (Al-Jabri et al., 2015; Campbell et al., 2014; Chen et al., 2014; Grace et al., 2015; Kim et al., 2011; Pereira et al., 2014).

Enjoyment comprises pleasure and flow (optimal psychological experience) and pleasure in the form of playfulness, fun and an intrinsic acceptance of social media (Hsu and $\mathrm{Wu}, 2011$; Kim et al., 2011; Wu and Chang, 2005; Zolkepli and Kamarulzaman, 2015). Selfenhancement consists of self-esteem (self-status and image), which enables users to gratify their outcome expectations of personal influences on social media. High self-enhancers have high self-esteem, due to which they overwhelmingly update and present their selfimage/status on social media and attract attention (Chen et al., 2014; Hennig-Thurau et al., 2004; Hepper et al., 2011; Hsu et al., 2007; Presi et al., 2014).

As such, it is found that users' engagement with social media is based primarily on personal intrinsic motivational factors that affect their behaviour in the use of social media. 


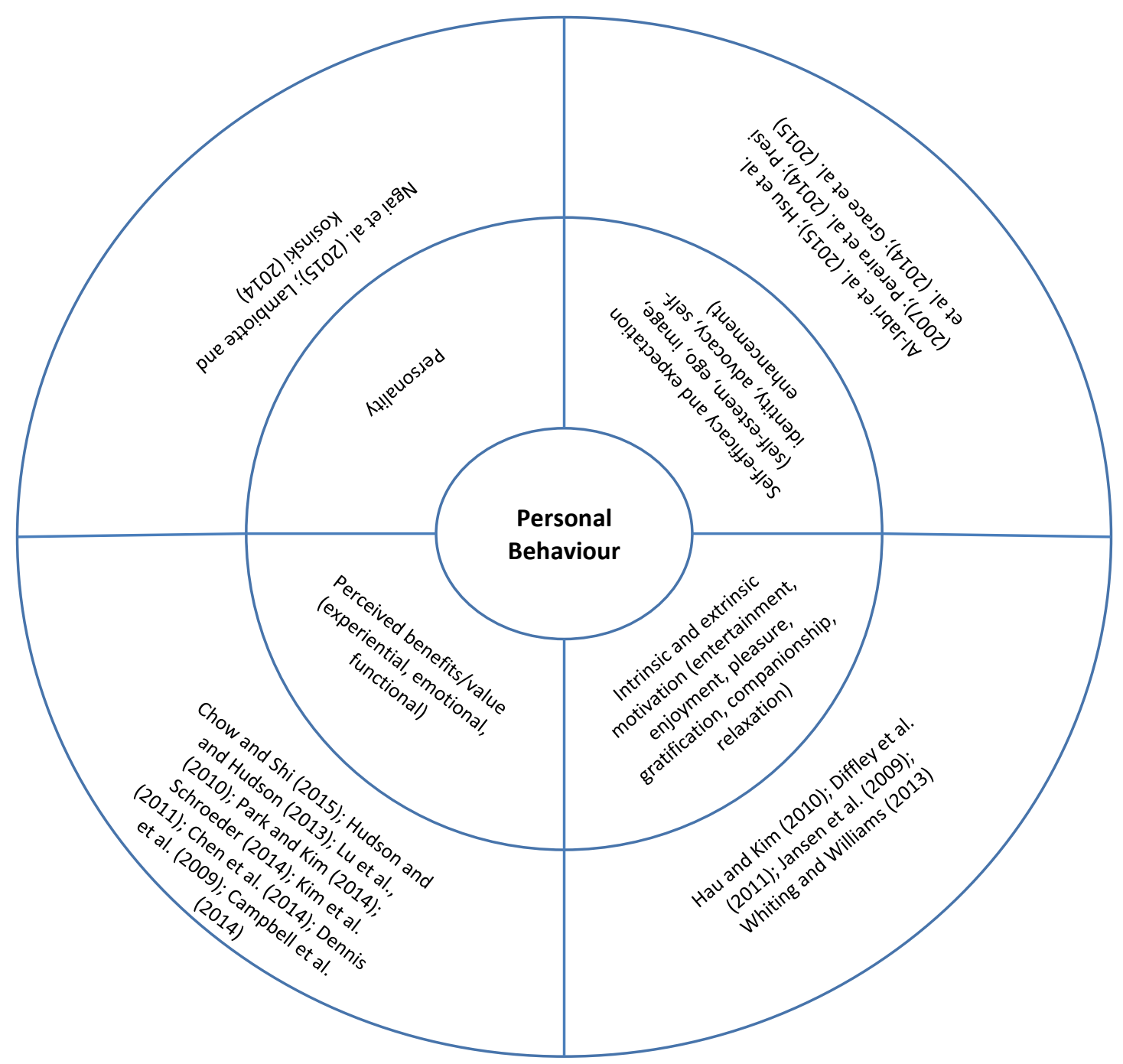

Fig. 2 Personal Behaviour

\section{Technology}

Technological factors are found to comprise performance expectancy (usefulness, utility), ease of use and relative advantage of the use of social media. Technological sub-factors are given in Figure 3. Performance expectancy is found to comprise perceived usefulness and utility of technology, which influences users' behavioural intentions. Similarly, ease of use includes convenience of the use of technology and relative advantage involves the innovative compatibility of social media over other technologies (Al-Gahtani et al., 2007; Lin and Anol, 2008; Venkatesh et al., 2003) and the prevailing adoption of technology (Carter and Weerakkody; 2008). It can be summarised that perceived usefulness, ease of use, relative advantage and innovation significantly influence social media users' behavioural intentions (Chiang, 2013; Hajli, 2014; Milewicz and Saxby, 2013; Pillai and Mukherjee, 2011; Zhang et al., 2014). Hence, technological factors which constitute social media usefulness, ease of use and compatibility, influence the intention to use of social media (Idemudia et al. 2016; Gironda and Korgaonkar, 2014; Lu et al., 2010; Wang et al., 2012; Zolkepli and Kamarulzaman, 2015). 


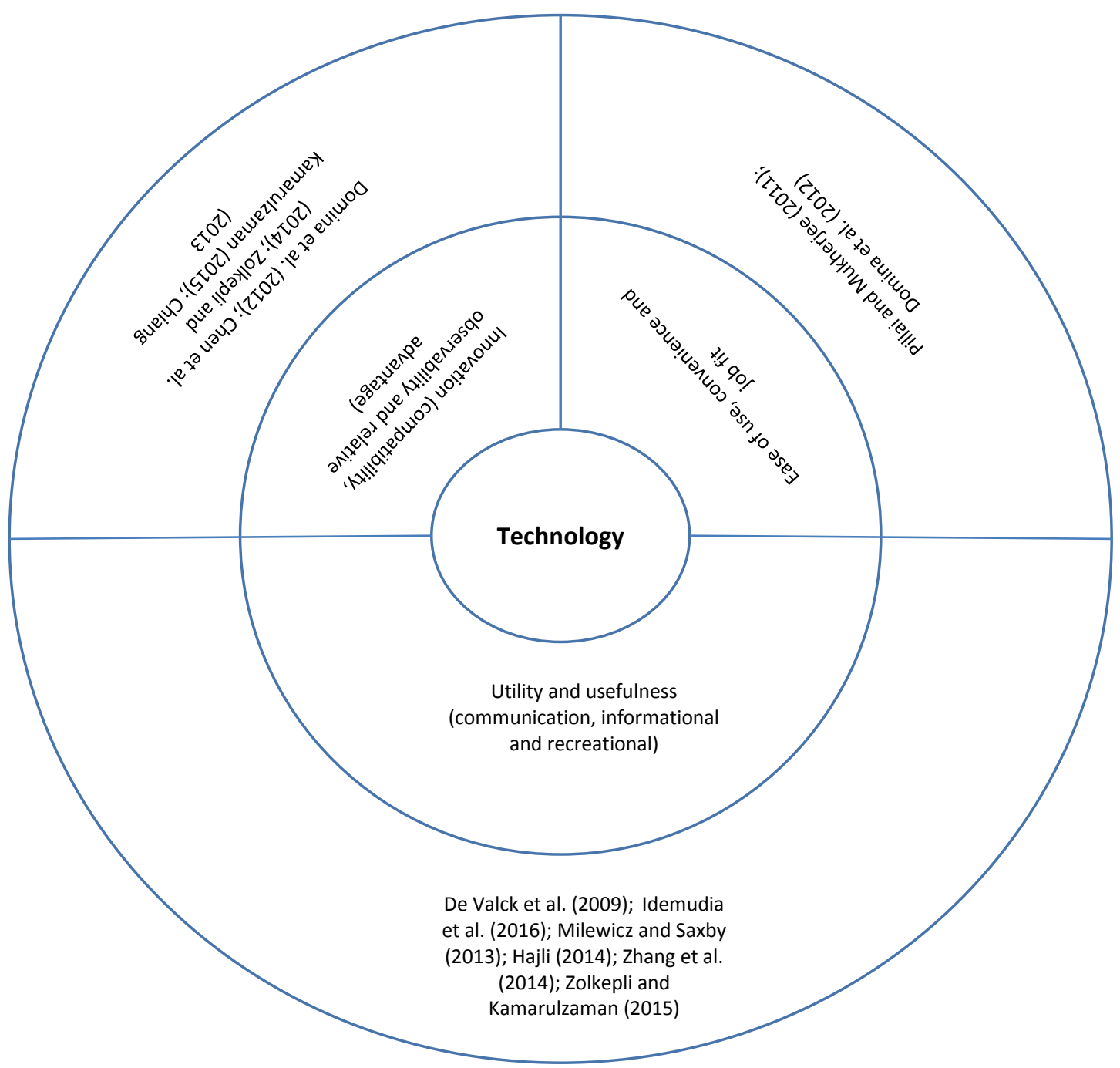

Fig.3 Technology

\section{Social factors}

Social factors include social interaction, social ties and social support (Bharati et al., 2014; Chiasson and Lovato, 2011; Grace et al., 2015; Talukder and Quazi, 2011; Venkatesh et al., 2003). These factors drive social interaction, which is a desire to connect, collaborate and communicate with others on social media (Chang and Chuang, 2011; De Valck et al., 2009; Hussain, 2012; Trivedi et al., 2106). Social Sub-factors are given in Figure 4.

Social interaction can be described as the desire to communicate, interact with others and build relationships on social media (Al-Jabri et al., 2015; Ko et al., 2005). Similarly, social media are perceived to enhance social interaction, connect people almost anywhere, give control over interaction and maintain social relations with others (family, friends etc.). It is a platform to release anxiety and depression, and to increase companionship and interpersonal utility, as suggested by the extant literature (Ellison et al., 2007; Grieve et al. 2013; Oldmeadow et al., 2013; Park et al., 2009; Whiting and Williams, 2013). Moreover, it is 
found that customers' pleasurable experience and peer pressure enhance social interaction on social media (Grace et al., 2015; Junglas et al., 2013).

Social ties denote building and maintaining relationships with other social media users (Rishika et al., 2013; Wang et al 2012). They are psychological goals of users to develop and maintain social relations with others, release anxiety and enhance interpersonal utility (Ellison et al., 2007; Grieve et al. 2013; Whiting and Williams, 2013), as it is human nature to socialise and interact with others (Dyson, 1998).

Similarly, social support includes willingness to help others and share anything that would assist others on social media (Liang et al., 2011; Zhang et al., 2014). It is a social aspect of exchange to share information with others (Ali, 2011; Crocker and Canevello, 2008) and also a major social value for social media users from other community members that use and interact with social media (Obst and Stafurik, 2010). As such, it can be summarised from the above findings that social factors (social interaction, social ties and social support) enhance the use of social media (Dalla Pozza, 2014; Foster et al., 2011; Gironda and Korgaonkar, 2014; Hsiao et al., 2010; Hsu and Wu, 2011).

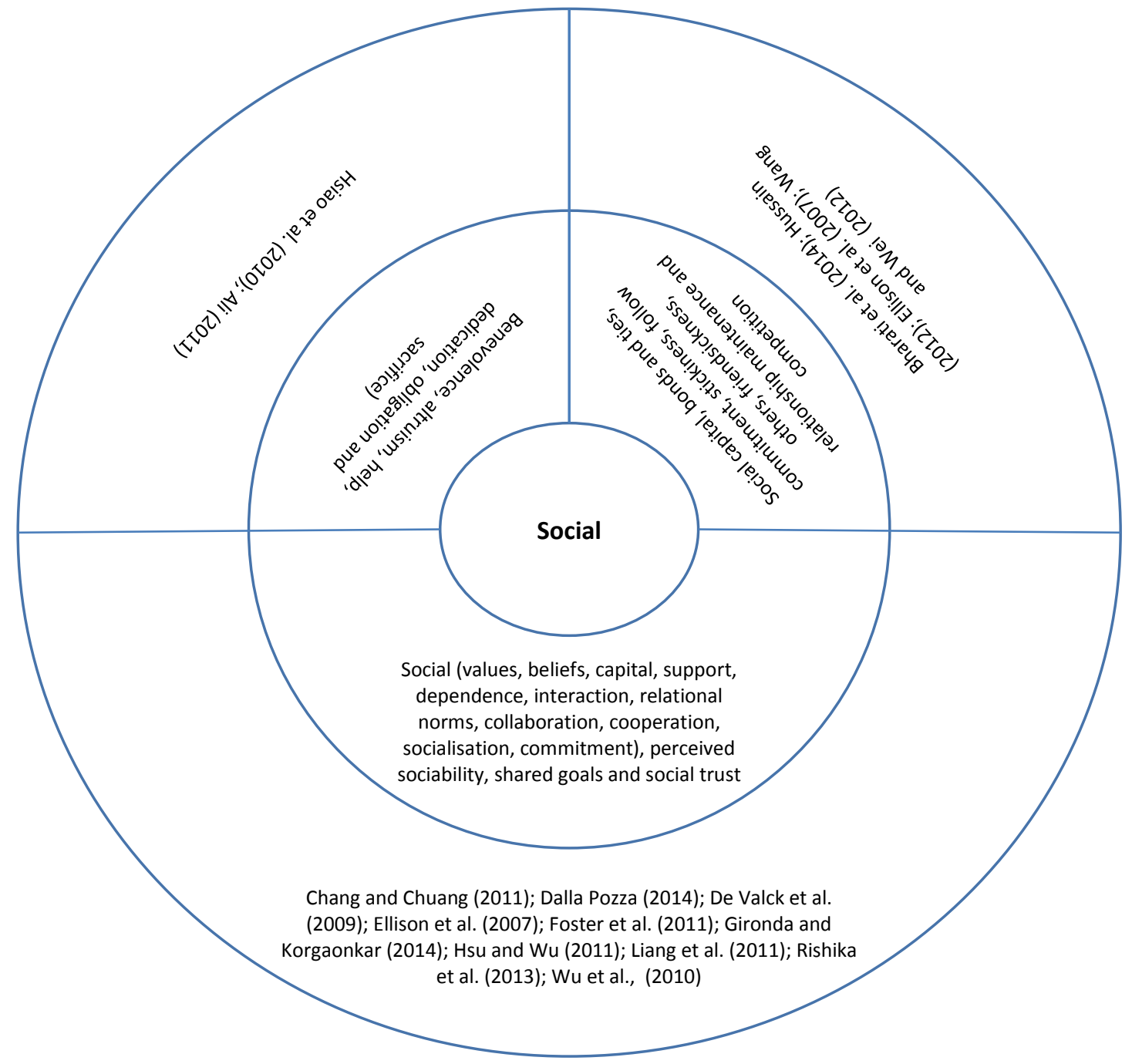

Figure $=4$ Social Factors 


\section{Privacy and Security}

Privacy and Security are found to comprise perceived risk, control and trust. Privacy involves users' willingness to share information online and the ability to control and choose to divulge personal information, whereas security relates to protection against the threat from the unauthorised access to personal information on social media (Belanger et al., 2002; Eastlick et al., 2006). Moreover, privacy also includes individuals' location, communication and information privacy (accumulation, treating and sharing information: Dinev et al., 2013). Sub-factors of privacy and security are given in Figure 5.

Privacy is described as users' perceived sense of risk regarding information about oneself and security is perceived as protection against the threat from unauthorised access to information about someone (Boyd, 2008; Lee et al. 2013). By joining and interacting with social media, users create their profiles, connect and share interests and personal information with others, which may potentially lead to personal privacy and security risks (Cheung et al., 2015; Tan et al., 2012).

Likewise, in existing literature trust has also emerged as one of the leading factors for privacy and security, and comprises users' confidence in the ability of a service provider to protect and monitor their personal information or reduce their uncertainty about the use of the service, as they also pay considerable heed to providers' (vendor and social media) integrity and benevolence (Cao et al., 2015; Cheung et al, 2015; Cheung and Lee, 2006; Krasnova et al., 2010). Trust is also noted to have a key relevance to social media users' perceived risk and it is developed through quality and source credibility (Burgess et al., 2011; Chen and Sharma, 2013; Gamboa and Gonçalves, 2014; Pentina et al., 2013; Shin, 2010; Zhou et al., 2016). From the above discussion, it is found that privacy and security can affect the use of social media and digital footprint generation. 


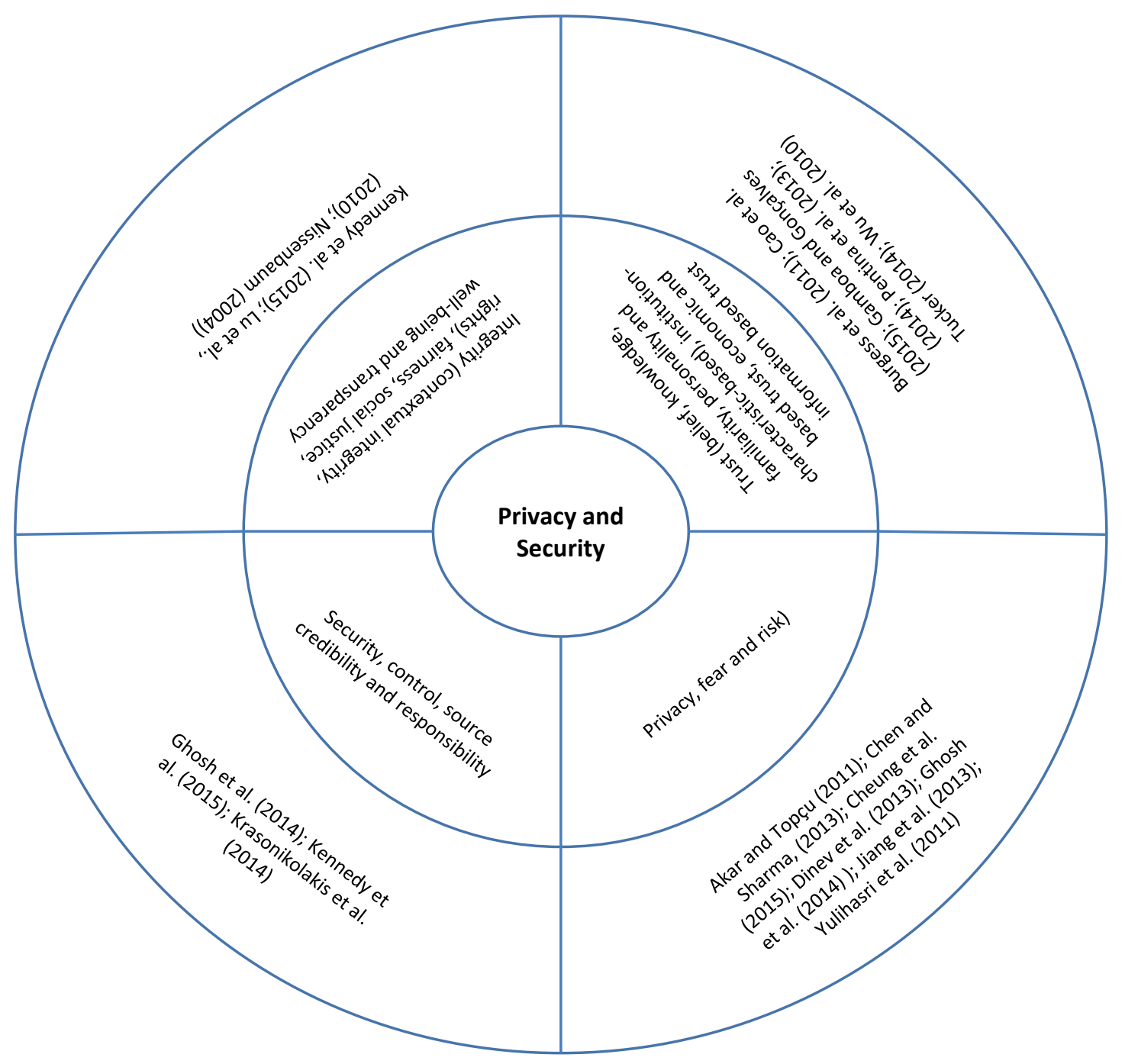

Fig.5 Privacy and Security

\section{Theoretical Foundation}

A diverse theoretical constructs have been used and applied in the existing literature to conceptualise the above issue. TAM, TRA, TPB and $U$ and $G$ theories have been most frequently used in examining customers' use and adoption of social media applications. More information has been provided in the Table- 1 .

\section{Research Approaches}

Positivist methods involving quantitative approaches and statistical testing appear to be more popular and common compared to interpretivist approach and/or qualitative methods. Quantitative research being a dominant method in this scholarship has used closed ended surveys. For model testing and data analysis, Structural Equation Modelling using AMOS and LISREL is a popular tool. For qualitative research, focus groups and semi-structured interviews were found to be the most widely used methods. Table 6 shows that 43 of the 58 
studies chose quantitative approaches, whereas 10 studies used qualitative approaches and only 5 studies chose mixed methods respectively.

Table 2 Methodological distribution

\begin{tabular}{|l|c|c|}
\hline \multicolumn{1}{|c|}{ Research Approach } & Number of studies & Percentage (\%) \\
\hline Quantitative & 43 & $74.14 \%$ \\
\hline Qualitative & 10 & $17.24 \%$ \\
\hline Mixed & 5 & $8.62 \%$ \\
\hline
\end{tabular}

Table 7 shows the distribution of journals, the number of articles from each journal and the percentage breakdown.

Table 3Source distribution

\begin{tabular}{|c|c|c|}
\hline Journal & Number of articles & Percentage $(\%)$ \\
\hline Information Systems Frontiers & 5 & $9 \%$ \\
\hline European Journal of Marketing & 5 & $9 \%$ \\
\hline Information \& Management & 4 & $7 \%$ \\
\hline Computers in Human Behaviour & 3 & $5 \%$ \\
\hline Business Horizons & 2 & $3 \%$ \\
\hline Decision Support Systems & 2 & $3 \%$ \\
\hline Information Systems Research & 2 & $3 \%$ \\
\hline Journal of Marketing Management & 2 & $3 \%$ \\
\hline Journal of Retailing and Consumer Services & 2 & $3 \%$ \\
\hline Online Information Review & 2 & $3 \%$ \\
\hline Big Data \& Society & 1 & $2 \%$ \\
\hline The International Journal of Research into New Media Technologies & 1 & $2 \%$ \\
\hline Electronic Commerce Research \& Applications & 1 & $2 \%$ \\
\hline Industrial Management \& Data Systems & 1 & $2 \%$ \\
\hline International Journal of Electronic Commerce & 1 & $2 \%$ \\
\hline International Journal of Event and Festival Management & 1 & $2 \%$ \\
\hline International Journal of Human-Computer Studies & 1 & $2 \%$ \\
\hline International Journal of Market Research & 1 & $2 \%$ \\
\hline International Journal of marketing studies & 1 & $2 \%$ \\
\hline International Journal of Virtual Communities and Social Networking & 1 & $2 \%$ \\
\hline Internet Research & 1 & $2 \%$ \\
\hline Irish Journal of Management & 1 & $2 \%$ \\
\hline Journal of Brand Management & 1 & $2 \%$ \\
\hline Journal of Business Research & 1 & $2 \%$ \\
\hline Journal of Computer-Mediated Communication & 1 & $2 \%$ \\
\hline Journal of Hospitality \& Tourism Research & 1 & $2 \%$ \\
\hline Journal of Indian Business Research & 1 & $2 \%$ \\
\hline Journal of Interactive Marketing & 1 & $2 \%$ \\
\hline Journal of Internet Commerce & 1 & $2 \%$ \\
\hline Journal of Management Information Systems & 1 & $2 \%$ \\
\hline Journal of Marketing Research & 1 & $2 \%$ \\
\hline Journal of Service Management & 1 & $2 \%$ \\
\hline Journal of the American society for information science and technology & 1 & $2 \%$ \\
\hline Management and Labour Studies & 1 & $2 \%$ \\
\hline Management Research Review & 1 & $2 \%$ \\
\hline Social and Behavioral Sciences & 1 & $2 \%$ \\
\hline Proceedings of the IEEE & 1 & $2 \%$ \\
\hline Qualitative Market Research: An International Journal & 1 & $2 \%$ \\
\hline The Journal of Computer Information Systems & 1 & $2 \%$ \\
\hline Total & 58 & $100 \%$ \\
\hline
\end{tabular}

\section{Conceptual Framework}

The current paper presents a conceptual framework (Figure 6) based on the relevant constructs for the antecedents of customers' willingness to generate and leave big data digital footprints on social media. The model is based on the Unified Theory of Acceptance and Use of Technology (UTAUT 2). UTAUT 2 is used because it takes into account various aspects 
of consumers' use of technology, such as motivation, innovation, technology utilisation and social aspects, as highlighted by the findings of this research. UTAUT 2 integrates elements on the use of technology from UTAUT 1 with similar themes (Venkatesh et al., 2012; Dwivedi et al., 2017). Hence, the proposed conceptual framework is developed by expanding on the UTAUT 2 model to examine the underlying factors that lead customers to generate and leave big data digital footprints on social media. The proposed framework has three antecedents of customers' behavioural intention (technological factors, social influence and personal behaviour), with privacy and security as moderator. From the findings delineated above, privacy and security are hypothesised to have moderating influence (facilitate or deter) on the relationship between customers' behavioural intention and its antecedents. Figure 6 presents the proposed framework and the following section explicates each component of the research model.

Figure 6 Conceptual Framework

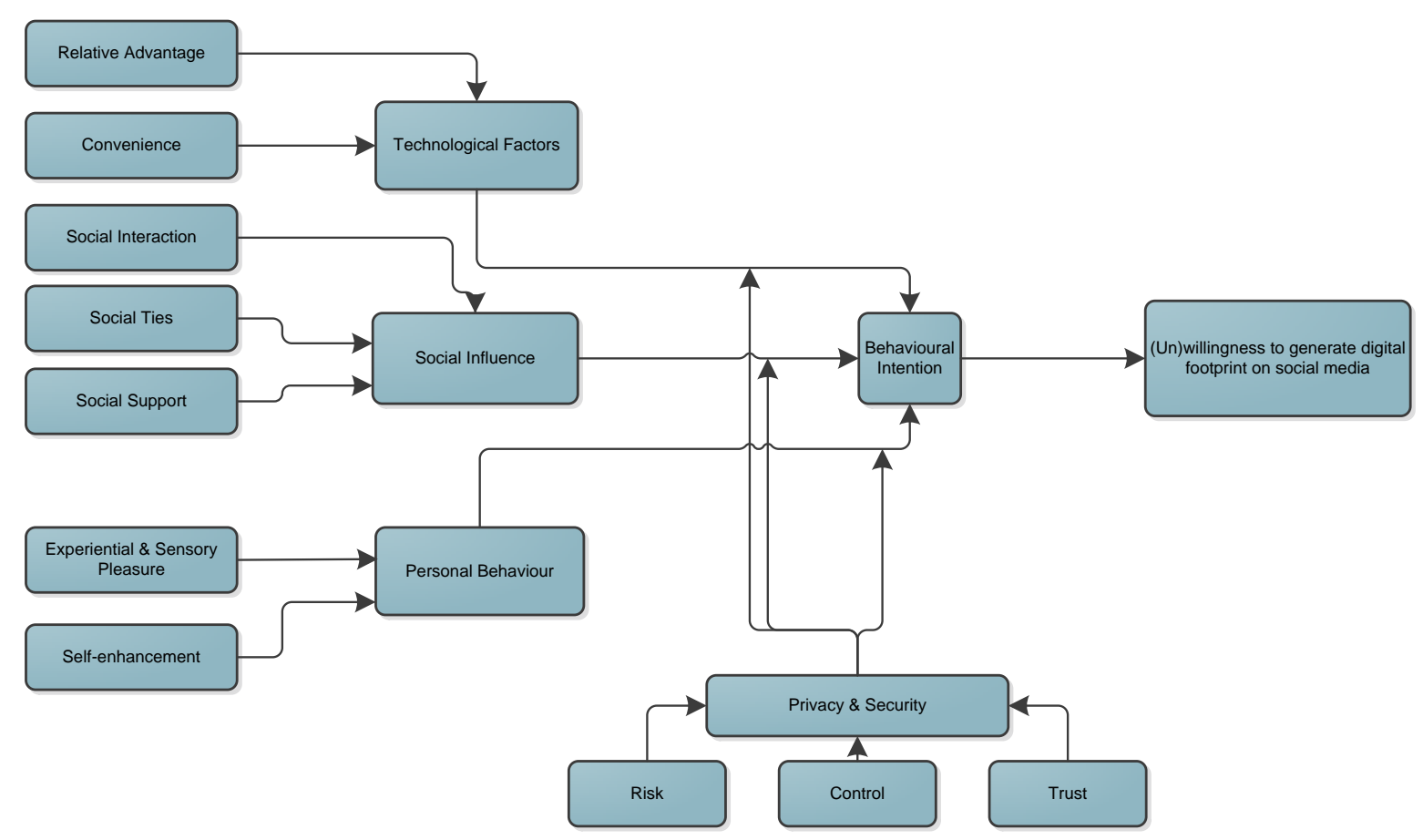

\section{Antecedent of customers' behavioural intention}

UTAUT 2 adopted from UTAUT 1 which unified the constructs for technology adoption in order to develop a more holistic understanding. The model suggests performance expectancy by combining similar themes such perceived usefulness, utility and relative advantage. Likewise, perceived ease of use and complexity are brought within effort expectancy; social norms, social factors and social image are combined for social influence; perceived behavioural control and compatibility are termed as facilitating conditions. UTAUT2 extends the model by integrating enjoyment within hedonic motivation, cost within price value and habit as determinants of customers' behavioural intention (Venkatesh et al., 2012). Some of the earlier works, such as Dwivedi et al. (2008) also ascertained some of these factors in their study of technology adoption, acceptance and diffusion indicating the validity of the model. The current paper has taken a similar stance and identified the following three antecedents of 
customers' behavioural intention in terms of their willingness to generate and leave digital footprints on social media. Details of each factor are given below. These factors, as delineated above, affect customers' digital footprint generation.

Technological factors: The systematic review of literature reveals that the technological factors in the form of usefulness, ease of use (convenience) and relative advantage can influence customers' interaction with social media and subsequent generation of digital footprints. Moreover, relative advantage comprises perceived technological innovation, which embodies convenience and compatibility, affecting customers' intention to use social media. Likewise, perceived usefulness was found to enhance job performance and to be instrumental in achieving valued outcomes, as suggested by the extant literature, such as Chen et al. (2009), Chiang (2013), Ho and Wu (2011), Lee et al. (2011) and Lin (2011). Hence, this study makes contribution by explicating the key technological factors of convenience and relative advantage that have a major influence on customers' big data digital footprint generation.

Social influence: This study has found that social influence is based on customers' perceived psychological social pressure, comprising social interaction, social ties and social support. This study contributes by unearthing the key social factors of social interaction, social ties and social support that hypothesised and tested to have influence on customers' psychological needs (Liang et al., 2011; Zhang et al., 2014), which result in big social data digital footprint generation on social media.

Personal behaviour: This study reveals two key personal behaviour factors of selfenhancement (self-efficacy and self-esteem) and perceived experiential and sensory pleasure benefits (hedonic and emotional; joy and enjoyment) that satisfy customers' hedonic needs (Hau and Kim, 2010; Park and Kim, 2014). Hence the paper makes a key contribution to the current scholarship by highlighting the key personal behavioural factors that determine customers' behavioural intention in generating big data digital footprints on social media.

Privacy and security: This study reveals that privacy and security comprise of perceived risks, control and trust. It has found that customers' self-perceived sense of risks in leaving personal information on social media and their sense of trust in social media providers (privacy) have a huge impact on their use of social media and their digital footprint generation. Equally, their perceived control and trust in service providers' ability and giving unauthorised access to others (security) affects their digital footprint generation on social media. This study, therefore, makes a contribution by revealing that customers' sense of privacy is enhanced when their perceived sense of risk increase and their perceived information control and perceived trust in social media decrease, which accordingly facilitates or deters digital footprint generation on social media.

\section{Theoretical contributions}

First of all, this research advances the literature on the factors that lead to customers' willingness to generate and leave big data digital footprints on social media. In doing so, it has extracted and analysed the key antecedents influencing customers' behavioural intentions in the form of technological factors, social influence and personal behaviour. It has also developed a model which exhibits the apposite and significant association among factors, providing valuable insights in determining customers' underlying behaviour in depositing big 
data digital footprints on social media, which can have practical implications for managers and practitioners. Furthermore, this study provides significant implications for the role of privacy and security for service providers (social media and vendors). Hence, the paper offers strong conceptual underpinning for assessing the dynamic and dichotomous nature of users' social media engagement. While, the model is suggested for social media based interaction, the privacy and security issue can also be applied for other technological applications such as cloud computing and smartphones.

\section{Conclusion}

This desk-based study has applied a systematic literature review of 501 peer-reviewed articles. The articles were scattered across 38 different journals, which substantiates that the area of research is of interest in many disciplines. However, marketing, international business and information systems are more in the limelight. This study has found that personal behaviour, technology, social influence and privacy and security are the key factors that influence customers' willingness to generate and leave big data digital footprints on social media. It has also developed a conceptual framework that offers deeper insights into the dynamics and kinetics of customers' social media use and their willingness to deposit digital DNA on these media. In addition, this study highlights the theories used profusely in previous scholarly works and articulates the research gap. Addressing the research gap not only advances and enriches the widely used technology adoption theories but can also facilitate future empirical works. A more holistic and robust understanding of consumers' ambivalence regarding the use of social media and their willingness (or a lack of it) of leaving digital footprints would enlighten future researchers and pave the way to more scholarly works in this field.

Nevertheless, the paper provides useful insights to the practitioners who can use the model to ascertain their target customers' motivation for and perceptions of using social media and other related interfaces. Business should realise that customers do not want to be chased; rather they would like to be wooed. Not all customers would have same motivation and/or similar level of desire to engage with social media. Hence, the social media providers such as Facebook and Instagram and the businesses who promote their products and services through these platforms and collect customer information should be aware of the sensitivities and intricacies pertaining to customers' privacy and security.

\section{Limitations and Future Research Directions}

There are some notable limitations of this study. Firstly, it has focused mainly on peerreviewed academic articles; future research could include monographs and industry reports. Secondly, the articles were clustered around the chosen four dimensions; future research could combine and choose the articles which have common factors. Thirdly, this study includes some articles around online social commerce; future research could focus just on social media customers. Last but not the very least, the conceptual framework offers a more generic model for assessing customers' willingness to leave digital footprints. Future research could include cultural factors to posit the model in specific socio-cultural contexts. 


\section{References}

Akar, E. and Topçu, B. (2011), "An examination of the factors influencing consumers' attitudes toward social media marketing", Journal of Internet Commerce, Vol. 10 No. 1, pp. 35-67.

Al-Gahtani, S. S., Hubona, G. S., and Wang, J. (2007). "Information technology (IT) in Saudi Arabia: culture and the acceptance and use of IT". Information and Management, 44(8), 681-691.

Al-Jabri, I.M., Sohail, M.S. and Ndubisi, N.O. (2015). "Understanding the usage of global social networking sites by Arabs through the lens of uses and gratifications theory." Journal of Service Management, Vol. 26, No.4, pp.662680.

Ali, H. (2011) "Exchanging value within individuals' networks: social support implications for health marketers." Journal of Marketing Management, Vol. 27, No. 3/4, pp. 316-335.

Baur, A. W. (2017). "Harnessing the social web to enhance insights into people's opinions in business, government and public administration." Information Systems Frontiers, 19(2), 231-251.

Bharati, P., Zhang, C., and Chaudhury, A. (2014). "Social media assimilation in firms: Investigating the roles of absorptive capacity and institutional pressures." Information Systems Frontiers, 16(2), 257-272.

Belanger, F., Hiller, J. S., and Smith, W. J. (2002). "Trustworthiness in electronic commerce: the role of privacy, security, and site attributes". The journal of strategic Information Systems, 11(3), 245-270.

Boyd, D. (2008). "Facebook's Privacy Trainwreck: Exposure, Invasion, and Social Convergence. Convergence". The International Journal of Research into new Media Technologies. 14(1), 13-20.

Burgess, S., Sellitto, C., Cox, C., \& Buultjens, J. (2011). "Trust perceptions of online travel information by different content creators: Some social and legal implications." Information Systems Frontiers, 13(2), 221-235.

Campbell, C., Ferraro, C. and Sands, S. (2014). "Segmenting consumer reactions to social network marketing." European Journal of Marketing, Vol.48, No.3/4, pp.432-452.

Cao, X., Guo, X., Liu, H., \& Gu, J. (2015). "The role of social media in supporting knowledge integration: A social capital analysis." Information Systems Frontiers, $17(2), 351-362$.

Carter, L., and Weerakkody, V. (2008). E-government adoption: A cultural comparison. Information Systems Frontiers, 10(4), 473-482. 
Chaffey, D. (2017). Global social media research summary 2017. [Web log comment]. Retrieved from: http://www.smartinsights.com/social-mediamarketing/social-media-strategy/new-global-social-media-research/.

Chang, H.H. and Chuang, S.S. (2011). "Social capital and individual motivations on knowledge sharing: Participant involvement as a moderator." Information and management, Vol. 48, No. 1, pp.9-18.

Charlesworth, A. (2014) An Introduction to Social Media Marketing. Oxon: Routledge.

Chen, J.V., Yen, D.C. and Chen, K. (2009), "The acceptance and diffusion of the innovative smartphone use: a case study of a delivery service company in logistics", Information and Management, 46 (4) 241-248.

Chen, A., Lu, Y., Chau, P.Y. and Gupta, S. (2014). "Classifying, Measuring, and Predicting Users' Overall Active Behavior on Social Networking Sites." Journal of Management Information Systems, Vol. 31, No. 3, pp. 213-253.

Chen, R., and Sharma, S. K. (2013). "Self-disclosure at social networking sites: An exploration through relational capitals." Information Systems Frontiers, 15(2), 269-278.

Cheung, C. M., and Lee, M. K. (2006). "Understanding consumer trust in Internet shopping: A multidisciplinary approach". Journal of the American society for Information Science and Technology, 57(4), 479-492.

Cheung, C., Lee, Z.W. and Chan, T.K. (2015). "Self-disclosure in social networking sites: the role of perceived cost, perceived benefits and social influence." Internet Research, Vol.25, No.2, pp.279-299.

Chiang, H.S. (2013). "Continuous usage of social networking sites: The effect of innovation and gratification attributes." Online Information Review, Vol. 37, No. 6, pp.851-871.

Chiasson, M. W., and Lovato, C. Y. (2001). "Factors influencing the formation of a user's perception and use of a DSS software innovation". Database for Advances in Information Systems, 32(3), 16-35.

Chow, W.S. and Shi, S. (2015). "Investigating customers' satisfaction with brand pages in social networking sites". The Journal of Computer Information Systems, Vol. 55, No.2, pp. 48.

Criteo (2016, June 20). A nation of social shoppers: 2.5 million Brits are buying on mobile every day, driven by social connections. [Web log comment]. Retrieved from http://www.criteo.com/news/

Crocker, J., and Canevello, A. (2008). "Creating and undermining social support in communal relationships: The role of compassionate and self-image goals". Journal of Personality and Social Psychology, 95 (3), 555-575. 
Cropanzano, R. (2009). "Writing nonempirical articles for Journal of Management: general thoughts and suggestions."

CSC. (2017, 01 May). Big data Universe beginning to explode. [Web log comment]. Retrieved from http://www.csc.com/insights/flxwd/78931big_data_universe_beginning_to_expl ode

Davis, F. D. (1989). "Perceived Usefulness, Perceived Ease of Use, and User Acceptance of Information Technology", M IS Quarterly, 13 (3), 319-339.

Dalla Pozza, I. (2014). "Multichannel management gets "social"." European Journal of Marketing, Vol.48, No.7/8, pp.1274-1295.

De Valck, K., van Bruggen, G.H. and Wierenga, B. (2009), "Virtual communities: a marketing perspective", Decision Support Systems, Vol. 47 No. 3, pp. 185203.

Dennis, C., Merrilees, B., Jayawardhena, C. and Tiu Wright, L. (2009). "Econsumer behaviour", European Journal of Marketing, Vol.43, No.9/10, pp.11211139.

Diffley, S., Kearns, J., Bennett, W. and Kawalek, P., (2011). "Consumer behaviour in social networking sites: implications for marketers." Irish Journal of Management, pp.47-66. Available at: http://iamireland.ie/wpcontent/uploads/2012/05/IJM_30_2_Final_crop.pdf\#page=57.

Dinev, T., Xu, H., Smith, J. H., and Hart, P. (2013). "Information privacy and correlates: an empirical attempt to bridge and distinguish privacy-related concepts", European Journal of Information Systems, 22(3), 295-316.

Domina, T., Lee, S.-E. and MacGillivray, M., (2012). "Understanding factors affecting consumer intention to shop in a virtual world", Journal of Retailing and Consumer Services, Vol. 19, No. 6, pp.613-620. Available at: http://linkinghub.elsevier.com/retrieve/pii/S0969698912000975.

Dwivedi, Y. K., Williams, M. D., and Venkatesh, V. (2008). "Guest Editorial: A profile of adoption of Information and Communication Technologies (ICT) research in the household context." Information Systems Frontiers, 10(4), 385390.

Dwivedi, Y. K., Rana, N. P., Jeyaraj, A., Clement, M., and Williams, M. D. (2017). "Re-examining the Unified Theory of Acceptance and Use of Technology (UTAUT): Towards a Revised Theoretical Model." Information Systems Frontiers, $1-16$.

DWork, C. and Mulligan, D. K. (2013). It's Not Privacy, and It's Not Fair. [Online] Available at: http://www.stanfordlawreview.org/online/privacy-and-big-data/itsnot-privacy-and-itsnot-fair [Accessed 28 June 2014]. 
Dyson, E. (1998). A Design for Living In the Digital Age, New York, NY, Broadway Books.

Eastlick M.A., Lotz S.L., Warrington P. (2006) "Understanding online B-to-C relationships: an integrated model of privacy concerns, trust, and commitment", Journal of Business Research, 59(8), 877-86.

Ellison, N.B., Steinfield, C. and Lampe, C. (2007). "The benefits of Facebook "friends:" Social capital and college students' use of online social network sites", Journal of Computer-Mediated Communication, Vol. 12, No.4, pp.1143-1168.

Fish, T. (2009). My Digital Footprint A two-sided digital business model where your privacy will be someone else's business!. futuretext.

Foster, M., West, B. and Francescucci, A. (2011). "Exploring social media user segmentation and online brand profiles", Journal of Brand Management, Vol. 19, No.1, pp.4-17.

Gamboa, A.M. and Gonçalves, H.M. (2014), "Customer loyalty through social networks: lessons from Zara on Facebook", Business Horizons, Vol. 57 No. 6, pp. 709-717.

Ghosh, A., Varshney, S. and Venugopal, P. (2014). "Social Media WOM: Definition, Consequences and Inter-relationships." Management and Labour Studies, Vol. 39, No. 3, pp.293-308.

Gilson, L. L., and Goldberg, C. B. (2015). Editors' comment: So, what is a conceptual paper?.

Gironda, J.T. and Korgaonkar, P.K. (2014). "Understanding consumers' social networking site usage", Journal of Marketing Management, Vol. 30, No.5-6, pp.571-605.

Grace, D., Ross, M. and Shao, W. (2015). "Examining the relationship between social media characteristics and psychological dispositions", European Journal of Marketing, Vol. 49, No. 9/10, pp.1366-1390.

Grieve, R., Indian, M., Witteveen, K., Anne Tolan, G. and Marrington, J. (2013). "Face-to-face or Facebook: can social connectedness be derived online?", Computers in Human Behaviour, 29 (3), 604-609.

Hajli, M.N. (2014). "A study of the impact of social media on consumers", International Journal of Market Research, Vol. 56 No. 3, pp. 388-404.

Hau, Y. S., \& Kim, Y. G. (2011). "Why would online gamers share their innovation-conducive knowledge in the online game user community? Integrating individual motivations and social capital perspectives." Computers in Human Behavior, 27(2), 956-970. 
Hepper, E.G., Hart, C.M., Gregg, A.P. and Sedikides, C. (2011). "Motivated expectations of positive feedback in social interactions", Journal of Social Psychology, 151(4), 455-477.

Hennig-Thurau, T., Gwinner, K.P., Walsh, G. and Gremler, D.D. (2004). "Electronic word-of-mouth via consumer-opinion platforms: what motivates consumers to articulate themselves on the Internet?", Journal of Interactive Marketing, 18(1), 38-52.

Ho, C., and Wu, W. (2011). "The role of innovativeness of consumer in relationship between perceived attributes of new products and intention to adopt", International Journal of Electronic Business Management, 9(3), 258266.

Hsiao, K.L., Lin, J.C.C., Wang, X.Y., Lu, H.P. and Yu, H. (2010), "Antecedents and consequences of trust in online product recommendations", Online Information Review, Vol. 34 No. 6, pp. 935-953.

Hsu, C. and Wu, C. (2011), "Understanding users' continuance of Facebook: an integrated model with the unified theory of acceptance and use of technology, expectation disconfirmation model, and flow theory", International Journal of Virtual Communities and Social Networking, Vol. 3 No. 2, pp. 1-16.

Hsu, C., Ju, T.L., Yen, C.H. and Chang, C.M. (2007). "Knowledge sharing behavior in virtual communities: The relationship between trust, self-efficacy, and outcome expectations." International Journal of Human-Computer Studies, 65(2), pp.153-169. Available at: http://linkinghub.elsevier.com/retrieve/pii/S1071581906001431.

Hudson, S. and Hudson, R. (2013). "Engaging with consumers using social media: a case study of music festivals." International Journal of Event and Festival Management, 4(3), pp.206-223. Available at: http://www.emeraldinsight.com/journals.htm?issn $=1758-$

2954andvolume $=4$ andissue $=3$ andarticleid $=17096678$ andshow $=\mathrm{html}$.

Hussain, I. (2012), "A study to evaluate the social media trends among university students", Procedia - Social and Behavioral Sciences, Vol. 64, pp. 639-645.

Idemudia, E. C., Raisinghani, M. S., \& Samuel-Ojo, O. (2016). "The contributing factors of continuance usage of social media: An empirical analysis." Information Systems Frontiers, 1-14.

Jansen, B.J., Zhang, M., Sobel, K. and Chowdury, A. (2009). "Twitter power: Tweets as electronic word of mouth." Journal of the American society for information science and technology, Vol 60, No. 11, pp.2169-2188.

Jiang, Z., Heng, C.S. and Choi, B.C. (2013). "Research Note-Privacy Concerns and Privacy-Protective Behavior in Synchronous Online Social Interactions." Information Systems Research, Vol.24 No.3, pp.579-595. 
Kane, G.C., Alavi, M., Labianca, G.J. and Borgatti, S. (2014). "What's different about social media networks? A framework and research agenda." MIS Quarterly, Vol. 38, No. 1, pp. 275-304

Junglas, I., Goel, L., Abraham, C. and Ives, B. (2013). "The social component of information systems - how sociability contributes to technology acceptance", Journal of the Association for Information Systems, 14 (10), 585-616.

Kennedy, H., Elgesem, D. and Miguel, C. (2015). "On fairness User perspectives on social media data mining." Convergence: The International Journal of Research into New Media Technologies, p.1354856515592507.

Kim, H.W., Gupta, S. and Koh, J. (2011). "Investigating the intention to purchase digital items in social networking communities: A customer value perspective." Information and Management, Vol. 48, No. 6, pp.228-234.

Ko, H., Cho, C.H. and Roberts, M.S. (2005). "Internet uses and gratifications: a structural equation model of interactive advertising", Journal of Advertising, 34 (2), 57-70.

Krasnova, H., Spiekermann, S., Koroleva, K., and Hildebrand, T. (2010). "Online social networks: why we disclose". Journal of Information Technology, 25(2), 109-125.

Krasonikolakis, I., Vrechopoulos, A. and Pouloudi, A. (2014), "Store selection criteria and sales prediction in virtual worlds", Information and Management, Vol. 51 No. 6, pp. 641-652.

Kuchler, H. (2017, April 4). The Internet of things: Home is where the hackers are. Financial Times. Retrieved from https://www.ft.com/content/cb880bc2057c-11e7-ace0-1ce02ef0def9

Lambiotte, R. and Kosinski, M. (2014). "Tracking the Digital Footprints of Personality." Proceedings of the IEEE, Vol.102, No.12, pp.1934-1939.

Lean, O. K., Zailani, S., Ramayah, T., and Fernando, Y. (2009). "Factors influencing intention to use e-government services among citizens in Malaysia", International Journal of Information Management, 29(6), 458-475.

Lee, H., Park, H., and Kim, J. (2013). "Why do people share their context information on Social Network Services? A qualitative study and an experimental study on users' behavior of balancing perceived benefit and risk", International Journal of Human-Computer Studies, 71(9), 862-877.

Lee, D., Park, J.Y., Kim, J. and Moon, J. (2011), "Understanding music sharing behaviour on social network services", Online Information Review, 35 (5), 716733.

Leung, X.Y., Bai, B. and Stahura, K.A. (2015). "The Marketing Effectiveness of Social Media in the Hotel Industry A Comparison of Facebook and Twitter". Journal of Hospitality and Tourism Research, Vol. 39, No.2, pp.147-169. 
Liang, T.-P., Ho, Y.-T., Li, Y.-W. ScTurban, E. (2011). "What drives social commerce: the role of social support and relationship quality." International Journal of Electronic Commerce, Vol. 16 No. 2 pp. 69-90.

Lin, H.F. (2011). "An empirical investigation of mobile banking adoption: the effect of innovation attributes and knowledge-based trust", International Journal of Information Management, 31 (3), 252-260.

Lin, C. P., and Anol, B. (2008). Learning online social support: an investigation of network information technology based on UTAUT. Cyberpsychology and Behavior, 11(3), 268-272.

Lu, Y, Zhao, L. and ScWang, B. (2010). "From virtual community members to c2c e-commerce buyers: trust in virtual communities and its effect on consumers' purchase intention." Electronic Commerce Research and Applications, Vol. 9 No. 4, pp. 346-360.

Malhotra, A., Totti, L., Meira Jr, W., Kumaraguru, P. and Almeida, V. (2012). "Studying user footprints in different online social networks." In Proceedings of the 2012 International Conference on Advances in Social Networks Analysis and Mining (ASONAM 2012), pp. 1065-1070, IEEE Computer Society.

Mathwick, C. (2002), "Understanding the online consumer: a typology of online relational norms and behavior", Journal of Interactive Marketing, Vol. 16 No. 1, pp. 40-55.

Michael, M.G., Michael, K. and Perakslis, C. (2014). "Uberveillance and the Internet of Things and people. In Contemporary Computing and Informatics (IC3I)," 2014 International Conference on (pp. 1381-1386). IEEE.

Milewicz and Saxby (2013). "Leaders' social media usage intentions for in-bound customer communications", Management Research Review, Vol. 36 No. 9 pp. 849-867.

Nill, A. and Schibrowsky, J.A. (2007). "Research on marketing ethics: A systematic review of the literature." Journal of Macromarketing, Vol.27, No.3, pp.256-273.

Nissenbaum, H., (2004). Privacy as contextual integrity. Wash. L. Rev., 79, p.119.

Ngai, E. W.T, Moon, K.K., Lam, S.S., Chin, E. S.K., and Tao, S.S.C (2015). "Social media models, technologies, and applications An academic review and case study", Industrial Management and Data Systems, Vol. 115 No. 5, pp.769802.

Ngai, E. W.T, and Wat, F.K.T (2002). "A literature review and classification of electronic commerce research", Information and Management, Vol. 39 No. 5, pp. 415-429.

Ngai, E.W.T., Xiu, L. and Chau, D.C.K. (2009), "Application of data mining techniques in customer relationship management: a literature review and 
classification", Expert Systems with Applications: An International Journal, Vol. 36 No. 2, pp. 2592-2602.

Park, N., Kee, K. and Valenzuela, S. (2009). "Being immersed in social networking environment: Facebook groups, uses and gratifications, and social outcomes", CyberPsychology and Behavior, 12 (6), 729-733.

Park, H. and Kim, Y.-K., (2014). "The role of social network websites in the consumer-brand relationship", Journal of Retailing and Consumer Services, 21(4), 460-467.

Pulse, G., (2012). Big Data for Development: Opportunities and Challenges: $A$ Global Pulse White Paper. [Online]

Available at: http://www.unglobalpulse.org/BigDataforDevWhitePaper [Accessed 1 May 2017].

Obst, P., and Stafurik, J. (2010). "Online we are all able bodied: Online psychological sense of community and social support found through membership of disability-specific Websites promotes well-being for people living with a physical disability", Journal of Community and Applied Social Psychology, 20 (6), 525-531.

Oldmeadow, J.A., Quinn, S. and Kowert, R. (2013). "Attachment style, social skills, and Facebook use amongst adults", Computers in Human Behaviour, 29 (3), 1142-1149.

Pentina, I., Zhang, L. and Basmanova, O. (2013), "Antecedents and consequences of trust in a social media brand: a cross-cultural study of Twitter", Computers in Human Behavior, Vol. 29, No. 4, pp. 1546-1555.

Pereira, H.G., de Fátima Salgueiro, M. and Mateus, I. (2014). "Say yes to Facebook and get your customers involved! Relationships in a world of social networks." Business Horizons, Vol. 57, No. 6, pp.695-702. Available at:http://www.sciencedirect.com/science/article/pii/S0007681314000937.

Pillai, A. and Mukherjee, J. (2011). "User acceptance of hedonic versus utilitarian social networking web sites." Journal of Indian Business Research, Vol. 3, No. 3, pp.180-191.

Presi, C., Saridakis, C. and Hartmans, S. (2014). "User-generated content behaviour of the dissatisfied service customer." European Journal of Marketing, Vol.48, No.9/10, pp.1600-1625.

Rauniar, R. Rawski, G. Yang, J. Johnson, B. (2013). "Technology acceptance model (TAM) and social media usage: an empirical study on Facebook", Journal of Enterprise Information Management, Vol. 27 No. 1, pp. 6-30. 
Rishika, R., Kumar, A., Janakiraman, R. and Bezawada, R. (2013). "The effect of customers' social media participation on customer visit frequency and profitability: an empirical investigation." Information systems research, Vol.24, No.1, pp.108-127.

Rosenberger, M., Lehrer, C., and Jung, R. (2017). "Integrating data from user activities of social networks into public administrations." Information Systems Frontiers, 19(2), 253-266.

Sayce, D. (2017, 29 April). Number of tweets per day. [Web log comment] Retrieved from: http://www.dsayce.com/social-media/tweets-day/.

Schroeder, R.(2014). "Big Data and the brave new world of social media research", Big Data and Society, Vol. 1, No. 2, p.2053951714563194.

Sharma, S. K. (2017). Integrating cognitive antecedents into TAM to explain mobile banking behavioral intention: A SEM-neural network modeling. Information Systems Frontiers, 1-13.

Shin, D. H. (2010). "The effects of trust, security and privacy in social networking: A security-based approach to understand the pattern of adoption", Interacting with computers, 22(5), 428-438.

Statista (2017). Statistics and facts about social media usage. Available at: https://www.statista.com/topics/1164/social-networks/. (accessed May 1, 2017).

Talukder, M., and Quazi, A. (2011). "The impact of social influence on individuals' adoption of innovation", Journal of Organizational Computing and Electronic Commerce, 21(2), 111-135.

Tan, X., Qin, L., Kim, Y., and Hsu, J. (2012). "Impact of privacy concern in social networking web sites", Internet Research, 22(2), 211-233.

Trivedi, N., Asamoah, D. A., and Doran, D. (2016). Keep the conversations going: engagement-based customer segmentation on online social service platforms. Information Systems Frontiers, 1-19.

Tucker, C.E. (2014). "Social networks, personalized advertising, and privacy controls." Journal of Marketing Research, Vol.51, No.5, pp.546-562.

Tuton, T. L. and Solomon, M. R. (2015) Social Media Marketing, $2^{\text {nd }}$ Edition, London: Sage Publication Ltd.

Venkatesh, V., Morris, M. G., Davis, G. B., and Davis, F. D. (2003). "User acceptance of information technology: toward a unified view", Management Information Systems Quarterly, 27(3), 425-478. 
Venkatesh, V., Thong, J. Y., and Xu, X. (2012). "Consumer acceptance and use of information technology: extending the unified theory of acceptance and use of technology."

Wang, X., Yu, C. and Wei, Y. (2012). "Social media peer communication and impacts on purchase intentions: a consumer socialization framework", Journal of Interactive Marketing, Vol. 26, No. 4, pp. 198-208.

Waters, R. and Bond, S. (2017, April 4). US moves step closer to overturning broadband privacy regulations. Financial Times. Retrieved from https://www.ft.com/content/fbb144fa-1411-11e7-80f4-13e067d5072c

Weick, K. E. (1989). "Theory construction as disciplined imagination." Academy of management review, 14(4), 516-531.

Whiting, A. and Williams, D (2013). "Why people use social media: a uses and gratifications approach", Qualitative Market Research: An International Journal, Vol. 16. No.4, pp.362-369.

Wu, J. J., and Chang, Y. S. (2005). "Towards understanding members' interactivity, trust, and flow in online travel community", Industrial Management and Data Systems, 105(7), 937-954.

Wu, J.-J., Chen, Y.-H. 8c Chung, Y.-S. (2010) "Trust factors influencing virtual community members: a study of transaction communities", Journal of Business Research, Vol 63, No. 9-10, pp. 1025-1032.

Yulihasri, Islam, M.A. and Daud, K.A.K., (2011). "Factors that influence customers' buying intention on shopping online", International Journal of marketing studies, Vol. 3, No. 1, pp.128.

Zhang, H., Lu, Y., Gupta, S. and Zhao, L. (2014). "What motivates customers to participate in social commerce? The impact of technological environments and virtual customer experiences", Information and Management, Vol. 51, No.8, pp.1017-1030.

Zhou, T., Lu, Y., and Wang, B. (2016). "Examining online consumers' initial trust building from an elaboration likelihood model perspective." Information Systems Frontiers, 18(2), 265-275.

Zolkepli, I.A. and Kamarulzaman, Y. (2015). "Social media adoption: The role of media needs and innovation characteristics", Computers in Human Behavior, Vol. 43, pp.189-209. 